\title{
Una herramienta de cálculo para la predicción de los Requerimientos de Capital en seguros de vida
}

\author{
SoníA GARCíA ${ }^{\mathrm{a}}$, José M. PAVÍA ${ }^{\mathrm{b}}$, ERNESTO J. Veres FERRER ${ }^{\mathrm{b}}$, JOSEP Lledó ${ }^{\mathrm{b}}$ \\ a Actuarial Services, Ernst \& Young, c/ Torre Picasso, 1, 28020 Madrid, España. E-mail: \\ gargarso@alumni.uv \\ ${ }^{b}$ Universidad de Valencia, Facultad de Economía, Avda. Tarongers, s/n, 46022 Valencia, España. E- \\ mail: pavia@uv.es, Ernesto.Veres@uv.es, jonabe@ulumni.uv.es
}

\begin{abstract}
RESUMEN
Solvencia II (Directiva parlamentaria europea 2009/138/EC) establece los criterios y normas de carácter cuantitativo y cualitativo que las entidades aseguradoras que operan en la Unión Europea deben acometer para garantizar su solvencia y estabilidad financiera. Solvencia II está estructurada en tres pilares y abarca, a través de un esquema modular, los distintos riesgos a los que está expuesta una entidad aseguradora. Este trabajo se centra en algunos de los submódulos del negocio de vida y estudia el Solvency Capital Requirement (SCR) para tres tipos de seguros: seguro de capital diferido, seguro de vida entera y seguro de renta inmediata. En concreto, analiza dentro del modelo estándar el cálculo del requerimiento de capital en los submódulos del riesgo de mortalidad y de longevidad y presenta una herramienta de cálculo muy sencilla para, prefijada la tabla de mortalidad, simplificar al máximo el cálculo del SCR de cualquier cartera de asegurados de estos submódulos. Se muestra cómo agrupando el total de pólizas de una cartera por edad del asegurado y/o temporalidad puede predecirse el capital requerido de solvencia para una cartera y un riesgo determinado a través de una función lineal. Al contrario de lo que cabría esperar por la ley de los grandes números, los requerimientos relativos de capital no decrecen con el tamaño de la cartera.
\end{abstract}

Palabras clave: SCR, Solvencia II, Gestión de riesgos.

\section{A tool for Predicting Solvency Capital Requirements in Life Insurance}

\begin{abstract}
Solvency II (European Parliament Directive 2009/138/EC) establishes the criteria and the quantitative and qualitative standards that insurance companies operating in the European Union must undertake to ensure their solvency and financial stability. Solvency II is structured in three pillars and covers, through a modular scheme, the various risks to which an insurer is exposed. This paper focuses on some of the life business modules and studies the Solvency Capital Requirement (SCR) for three types of insurances: endowment policy, whole life insurance and immediate renters insurance. In particular, the work analyzes, within the standard model, the calculus of the capital requirement for the mortality and longevity sub-modules and presents a very simple tool that, given the life table, simplifies SCR operations for any single policy of these sub-modules. The analysis shows how the SCR for a portfolio may be predicted through a linear function by grouping policies by age and/or timing. Contrary to what would be expected from the law of large numbers, relative capital requirements do not decrease with the size of the portfolio.
\end{abstract}

Keywords: SCR, Solvency II, Risk Management.

Clasificación JEL: G22, G23, G17

Artículo recibido en febrero de 2017 y aceptado en abril de 2017

Artículo disponible en versión electrónica en la página www.revista-eea.net, ref. ə-35207

ISSN 1697-5731 (online) - ISSN 1133-3197 (print) 


\section{INTRODUCCIÓN: POR QUÉ DE SOLVENCIA II}

Es comúnmente aceptado que la magnitud fundamental a la que ha de atender la gestión de toda entidad aseguradora es su solvencia. Debido a su función mediadora en el sistema financiero, las aseguradoras recogen el ahorro presente de los asegurados para indemnizar en el futuro a aquellos que sufran las consecuencias desfavorables de un riesgo asegurado. En el mundo asegurador, un mundo repleto de riesgos, se ha de procurar mantener mercados eficientes, justos, firmes y estables que garanticen la protección de los asegurados.

La Comisión Europea legisló en su momento un sistema de ratios (Solvencia I), basados tanto en el nivel de siniestralidad como en el nivel de primas, como mecanismo de salvaguarda ante los efectos que los cambios en las principales variables económicas, financieras o actuariales pudieran provocar en el sistema asegurador (Sandström, 2006). Este conjunto de ratios han sido aplicados de la misma forma por todos los aseguradores, fuera cual fuese el tamaño de la entidad y el perfil de sus operaciones. Es lógico pensar, sin embargo, que la solvencia de una aseguradora no debería estimarse únicamente a partir de datos financieros que surgen de un balance contable y que deberían tenerse en cuenta también otros aspectos, como la exposición al riesgo; siendo importante además identificar los riesgos que asume cada aseguradora y la gestión que efectúa de los mismos.

A pesar de lo anterior, Solvencia I no contempla los perfiles de riesgo de cada entidad y su aplicación da lugar a situaciones poco comprensibles. Por ejemplo, consideremos dos entidades que, teniendo el mismo volumen de reservas y de primas, actúan con políticas de inversión muy diferentes: una de forma prudente y la otra de forma agresiva. Para Solvencia I los recursos propios para ambas entidades deberían ser similares. Pero no parece que esto sea lógico, en cuanto que la entidad de política agresiva soporta más riesgo que la otra, por lo que se le debería exigir recursos propios superiores que la entidad que actúa prudentemente.

Según Camacho (2009, pág. 4), “el creciente proceso de globalización y creación de un mercado financiero único requiere la homogeneización de los criterios establecidos por los estados miembros de la Unión Europea para la medición de los niveles de solvencia de las entidades aseguradoras", motivo que ha propiciado la necesidad de modificar el modelo europeo de solvencia, que ha culminado con Solvencia II. A fin de perfeccionar los sistemas de medición y de control, la Comisión Europea propuso en su Directiva 2009/138/EC el proyecto Solvencia II. ${ }^{1}$

En un cambio de orientación, Solvencia II está enfocada a que cada empresa aseguradora conozca cuál es su posicionamiento frente a los distintos riesgos

\footnotetext{
${ }^{1}$ http://eur-lex.europa.eu/JOHtml.do?uri=OJ:L:2009:335:SOM:EN:HTML
} 
que está asumiendo y a exigir la adecuada gestión de los mismos (Alonso y Albarrán, 2008), incluyendo las actuaciones y previsiones a llevar a cabo con el fin de implantar en el sector asegurador los principios orientadores y la búsqueda de procedimientos para el cálculo de unos requerimientos de capital acordes con el nivel de riesgo gestionado.

La Directiva que recoge Solvencia II está estructurada bajo el principio de los tres pilares (ver, por ejemplo, Ferri, 2012) ${ }^{2}$ y tiene como eje central la correspondencia entre capital requerido y riesgos asumidos, para lo que es absolutamente necesaria una evaluación adecuada de los mismos centrada en la defensa del asegurado. Según UNESPA ${ }^{3}$ la adopción del proyecto Solvencia II por parte de los países miembros de la Unión Europea introduce un nuevo sistema de solvencia caracterizado por un enfoque ajustado al riesgo, que permite establecer un requerimiento de capital alineado con el perfil de riesgos de cada compañía de seguros. Al vincular la gestión global de riesgos con el nivel de fondos propios necesarios, la implantación de Solvencia II tiene una incidencia directa sobre la rentabilidad de las entidades, incrementará la transparencia del sector, aumentará la eficiencia en el uso del capital y conseguirá una mejora en la gestión empresarial (Albarrán y Lozano, 2010).

Recientes trabajos van más allá e incorporan modelizaciones y nuevas técnicas de estimación del Solvency Capital Requirement (SCR) respetando las especificaciones de Solvencia II. Por ejemplo, Christiansen y Niemeyer (2014) ponen el énfasis en proporcionar una definición sólida del margen de riesgo y comparan, mediante un modelo matemático, diferentes interpretaciones para la definición del SCR. Fröhlich y Weng (2015) se centran en la correcta estimación de la posible pérdida de fondos propios básicos durante el siguiente ejercicio económico y, basándose en ideas estrechamente relacionadas con el concepto de inferencia fiducial, introducen un nuevo enfoque de la incertidumbre asociada a los parámetros del modelo.

En este trabajo utilizaremos indistintamente el término inglés para Solvency Capital Requirement (SCR) y los términos españoles equivalentes Capital de Solvencia Obligatorio, Requerimientos de Capital y Requerimientos de Capital Solvencia.

Aproximaciones al problema de la solvencia y medición del riesgo en otros sectores económicos y perspectivas han sido elaboradas por diversos autores.

\footnotetext{
${ }^{2}$ El primer Pilar hace referencia al cálculo de la cifra de capital necesaria para hacer frente a los riesgos asumidos por la compañía. El Pilar II se centra en lo que será la actuación de los supervisores dentro de Solvencia II. Y el Pilar III trata de fomentar la disciplina de mercado exigiendo que se facilite información relevante respecto a la adecuación del capital de las aseguradoras.

3 La Asociación Empresarial del Seguro (UNESPA) representa a cerca de 240 entidades aseguradoras lo que supone algo más del 96\% del mercado asegurador español.
} 
Abad y Gutiérrez (2014) evalúan la solvencia bancaria mediante un modelo de regresión múltiple en el que las variables explicativas son ratios extraídos de los estados contables públicos. La medición del riesgo soberano y solvencia país, mediante un modelo con datos de panel, es el objeto de atención del trabajo de García y Vicens (2006); mientras que Fruet-Cardozo, Cañas-Madueño y Millán de la Lastra (2014) analizan los criterios del riesgo-país para determinar la Prima de Riesgo. Por su parte, Rosado y Alonso (2015) estudian el efecto que sobre la solvencia y la suficiencia de las pensiones públicas y privadas han tenido tanto la reforma del sistema de pensiones español como el tratamiento fiscal del ahorro de 2014.

Como Solvencia II es muy amplia y abarca los distintos riesgos a los que se expone una entidad aseguradora, este trabajo se centra en el negocio de vida y, más concretamente, en el riesgo de mortalidad y en el riesgo de longevidad y estudia los requerimientos del capital de solvencia dentro del denominado modelo estándar para tres tipos de seguros: seguro de capital diferido, seguro de vida entera y seguro de renta inmediata. La finalidad perseguida es la construcción de una herramienta predictiva que simplifique al máximo el cálculo (la estimación) del SCR de una determinada cartera de asegurados, dada una tabla de mortalidad prefijada.

\section{SOLVENCY CAPITAL REQUIREMENT}

Los requerimientos del capital de solvencia se pueden definir como la cantidad de fondos propios necesarios para hacer insignificante la probabilidad de ruina de la aseguradora en el plazo de un año (Pozuelo de Gracia, 2007). El SCR es un requisito basado en el riesgo soportado por la aseguradora que garantiza un mínimo de capital para mantener la protección apropiada a los asegurados y la estabilidad del mercado. Es necesario predecir el montante de esta cantidad, lo que puede hacerse bien mediante el uso del modelo estándar común para todas las entidades y en el cual se basa el método predictivo que desarrollaremos a lo largo del trabajo y, o bien mediante el uso de modelos internos, que requieren una autorización previa a su utilización por parte del supervisor.

El modelo estándar consiste en un conjunto de fórmulas y metodologías propuestas por el regulador con las que la entidad aseguradora puede anticipar el correspondiente capital de solvencia (SCR). El CEIOPS ${ }^{4}$ ha elaborado un modelo de SCR siguiendo un esquema piramidal (ver Figura 1). En él se

\footnotetext{
${ }^{4}$ The Committee of European Insurance and Occupational Pensions Supervisors (CEIOPS) está compuesto por figuras representativas de las autoridades supervisoras de seguros y pensiones de los estados miembros de la Unión Europea y está encargado de desarrollar Solvencia II. Dicho Comité ha sido sustituido desde el 1 de enero de 2011 por la European Insurance and Occupational Pensions Authority (EIOPA).
} 
identifican los distintos riesgos a los cuales se enfrentan las entidades aseguradoras para, de esta forma, poder estimar de una manera sistemática el futuro requerimiento de capital global de cada una de ellas. El QIS5 ${ }^{5}$, que es el quinto de una línea de estudios de impacto cuantitativo desarrollados entre 2006 y 2010 por CEIOPS, se ha utilizado para desarrollar la fórmula estándar para todas las aseguradoras de la UE que no utilizan un modelo interno previamente aprobado. La determinación del Solvency Capital Requirement se debe realizar teniendo en cuenta los principales riesgos de la actividad aseguradora y la aproximación de la pérdida máxima posible que en su conjunto podría manifestarse en un horizonte temporal de un año con una probabilidad de un 99,5\%. Es de destacar que con la estimación del SCR se pretenden cubrir las pérdidas inesperadas puesto que las esperadas ya deben estar adecuadamente recogidas en el valor de las reservas técnicas (Camacho, 2009).

El SCR tiene una estructura piramidal en la que los módulos y submódulos que lo componen no son independientes entre sí, con lo que existe una matriz de correlación para cada nivel de la pirámide. Estas matrices son uno de los aspectos clave para la predicción del montante del SCR global (Ferri, 2012), pues delimitan su valor final al combinar los requerimientos de cada riesgo. En concreto, la estructura de cálculo del SCR global viene determinada por la expresión:

$$
S C R=B S C R+S C R_{o p}+A d j
$$

dónde, $S C R$ representa el capital obligatorio global en la fórmula estándar, $B S C R$ denota el capital de solvencia obligatorio básico, $S C R_{o p}$ simboliza el gasto de requerimiento de capital para el cálculo operacional y $A d j$ es el ajuste por el efecto de absorción de riesgo de las provisiones técnicas e impuestos diferidos.

El cálculo del Capital de Solvencia Obligatorio Básico (BSCR) surge de la predicción combinada de los requerimientos de capital de los seis módulos de riesgo -riesgo de mercado, riesgo de suscripción de salud, riesgo de impago de la contraparte, riesgo de suscripción de vida, riesgo de suscripción de no vida, y riesgo de los activos intangibles (ver nivel tres de la Figura 1)-, cada uno de los cuales es una agregación de sus correspondientes submódulos que tiene en cuenta su estructura de correlaciones, y su fórmula habitual quedaría como:

$$
B S C R=\sqrt{\sum_{i, j} \operatorname{Corr}_{i j} \cdot S C R_{i} \cdot S C R_{j}}+S C R_{\text {intangibles }}
$$

donde $\operatorname{Corr}_{i j}$ es el coeficiente de correlación entre los riesgos $i$ y j.

\footnotetext{
${ }^{5}$ https://eiopa.europa.eu/consultations/qis/insurance/quantitative-impact-study-5/index.html
} 
Figura 1

Estructura modular para el cálculo del SCR en el modelo estándar

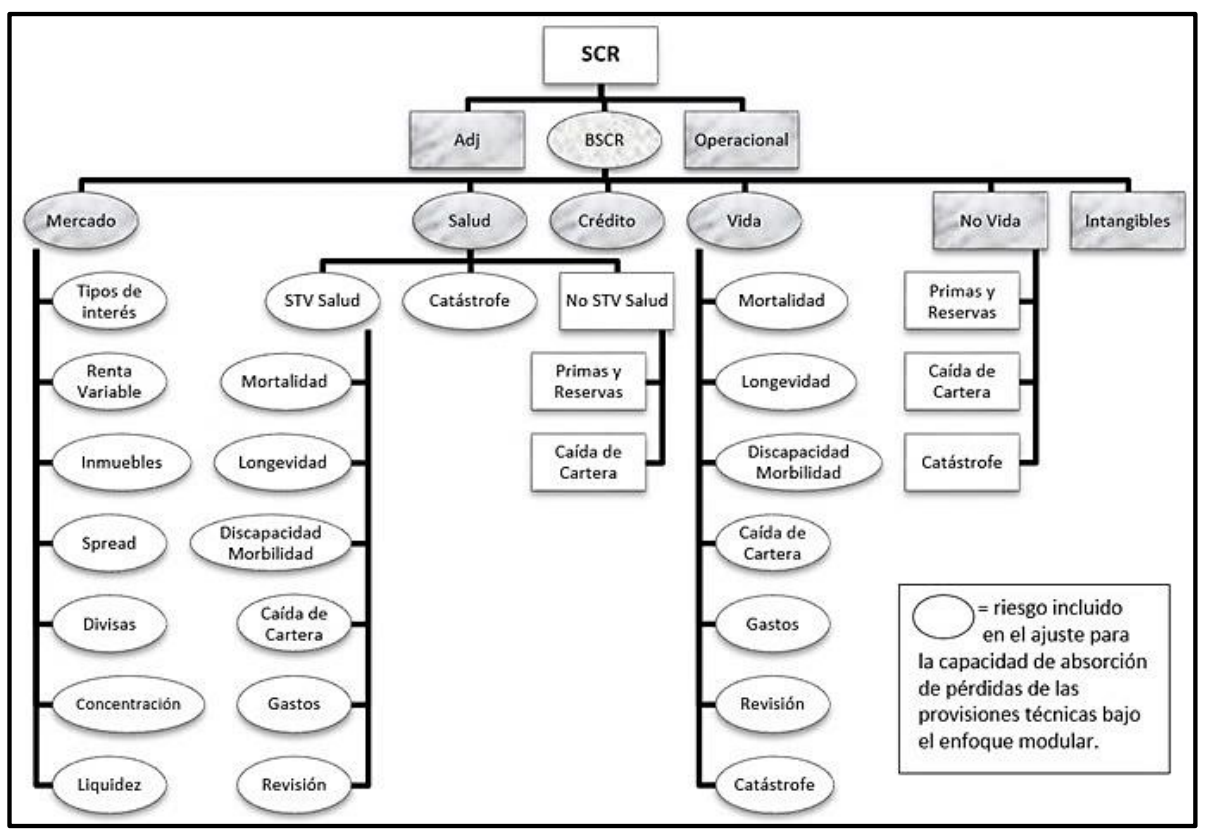

Fuente: Elaboración propia a partir de Comisión Europea (2010, p. 90)

En el cálculo predictivo del SCR para cada módulo (y submódulo), las entidades calculan un balance en condiciones de estrés, y lo comparan con el balance no estresado que han empleado para valorar sus fondos propios. Por tanto para cada submódulo la entidad debe obtener el valor del best estimate ${ }^{6}$ de las provisiones técnicas vinculadas. La diferencia resultante de las provisiones mide el impacto de la mitigación del riesgo.

\section{SCR DEL RIESGO DE VIDA}

El riesgo de suscripción de vida, según las especificaciones del QIS5, está asociado tanto a los riesgos cubiertos como a los procesos que se siguen en el ejercicio de la actividad. Los cálculos del capital de solvencia en este módulo se basan en escenarios específicos. En este caso, el capital obligatorio se define como el impacto de un escenario dado sobre el valor neto de los activos de la entidad y se calcula como activos menos pasivos, de los que se excluyen los pasivos subordinados. Conforme lo especificado en la Directiva Solvencia II,

\footnotetext{
${ }^{6}$ La media ponderada por la probabilidad de los flujos de efectivo futuros, teniendo en cuenta el valor del dinero en el tiempo y usando una estructura temporal de la tasa de interés libre de riesgo relevante.
} 
esta medida garantiza que el valor neto de los activos responda realmente a los fondos propios básicos, es decir, el excedente de activos sobre pasivos más los pasivos subordinados.

Un primer escenario consiste en un estrés inmediato que ocurre en la fecha de valoración y el capital requerido sería la pérdida inmediata en fondos propios básicos resultante al aplicar dicho estrés. Hay que tener en cuenta que los escenarios no recogen las variaciones de activos y pasivos en los 12 meses siguientes a la situación de estrés.

El módulo de riesgo de suscripción de vida se compone de siete submódulos (ver Figura 1), cuya definición/valoración, siguiendo a Camacho (2009), se concreta en ${ }^{7}$ :

a) Riesgo de mortalidad. Es el riesgo de que la mortalidad de los asegurados de la compañía sea mayor que la esperada a través de las tablas de mortalidad utilizadas. Su valoración se realiza mediante pruebas de estrés, presuponiendo incrementos en las tasas de mortalidad del 15\%. La variación de las reservas producto de cambios en las tasas de mortalidad determina los requerimientos de capital para este riesgo.

b) Riesgo de longevidad. Es el riesgo de que la supervivencia de los asegurados de la compañía sea mayor que la esperada como consecuencia de cambios en las tasas de mortalidad. Su determinación se realiza mediante pruebas de estrés, presuponiendo decrementos en las tasas de mortalidad del $20 \%$. El capital de solvencia para el riesgo de longevidad se identifica con el cambio en las provisiones debido al shock en las tasas de mortalidad.

c) Riesgo de invalidez. Es el riesgo de que la invalidez de los asegurados de la compañía sea mayor que la esperada a través de las tablas de invalidez utilizadas, producto de cambios en las tasas de invalidez. Su valoración se realiza mediante pruebas de estrés, presuponiendo un incremento en la tasa global de invalidez del 35\%, en el primer año y del 25\% para todas las edades en los años subsiguientes. Los cambios en las tablas de invalidez una vez aplicado el shock son los que determinan el SCR para el riesgo de invalidez.

d) Riesgo de cancelación o rescate. Es el riesgo de que las tasas de cancelaciones de las pólizas difieran de las tasas de cancelaciones esperadas por la compañía, e impacten en el patrimonio de la aseguradora. Su determinación se realiza mediante pruebas de estrés, estimando variaciones en las tasas de cancelaciones. Según se especifica en el QIS5 el capital de solvencia para el riesgo de caída de la cartera viene determinado

\footnotetext{
${ }^{7}$ La transcripción literal de los distintos riesgos se puede consultar en la Directiva 2009/138/CE (Parlamento Europeo y Consejo, 2009).
} 
por el mayor de los siguientes tres escenarios: a) riesgo de rescate en caso de disminución de las tasas de rescate al 50\%; b) riesgo de rescate en caso de aumento de las tasas de rescate en un $50 \%$; c) riesgo de rescate suponiendo un evento de rescate masivo ${ }^{8}$. QIS5 propone determinar los tres requerimientos de capital para el riesgo de rescate y elegir el mayor.

e) Riesgo de gastos. Es el riesgo de que las tasas de gastos establecidos en los seguros de vida difieran de los gastos esperados e impacten negativamente en el patrimonio de la empresa. Su valoración se realiza mediante pruebas de estrés, aumentando las tasas de gastos en un $10 \%$ y las tasas de inflación referida a los gastos en forma incremental un $1 \%$ anual.

f) Riesgo de revisión. Es el riesgo de pérdida o de minusvaloración del pasivo vinculado a seguros y reaseguros debido a cambios en el entorno legal o en el estado de salud del asegurado. Su valoración se realiza incrementando en un $3 \%$ la cantidad anual pagadera por anualidades sujetas a este riesgo. El impacto debe evaluarse considerando exclusivamente los pagos pendientes.

g) Riesgo de catástrofe. Es el riesgo de que un evento irregular y que no esté suficientemente recogido en los otros submódulos, afecte el patrimonio de la empresa. Este riesgo está asociado a riesgos extremos o eventos que no se encuentran adecuadamente recogidos en los otros submódulos, como por ejemplo una pandemia. Este tipo de riesgo se calcula mediante un incremento absoluto del 1,5 por mil en la tasa en que fallecen los asegurados a lo largo del año siguiente, solo aplicables a pólizas dependientes de la mortalidad.

Una vez determinados los requerimientos de capital para cada uno de los submódulos, corresponde determinar el requerimiento de capital para el riesgo vida, que se determina, de modo similar al BSCR, como la suma de los SCR de los correspondientes submódulos teniendo en cuenta las correlaciones existentes entre ellos: ${ }^{9}$

$$
S C R_{\text {life }}=\sqrt{\sum_{r, c} \text { CorrLife }_{r, c} \cdot S C R_{r} \cdot S C R_{c}}
$$

dónde CorrLife ${ }_{\mathrm{r}, \mathrm{c}}$ son los coeficientes de correlación entre los riesgos $r$ y $c$, $\mathrm{SCR}_{\mathrm{r}}$ y $\mathrm{SCR}_{\mathrm{c}}$ representan los requerimientos de capital para los riesgos $r$ y $c$ del módulo de vida y $\mathrm{SCR}_{\text {life }}$ simboliza el requerimiento total de capital por riesgo de suscripción de vida.

\footnotetext{
${ }^{8}$ El rescate masivo consta de la combinación de los siguientes cambios: (i) rescate del 30\% de las pólizas de seguro con limitación positiva de rescate distintas a negocio de colectivos; y, (ii) rescate del 70\% de las pólizas de seguro con limitación positiva de rescate para negocio de colectivos.

${ }^{9}$ Las correlaciones propuestas en el modelo estándar para los submódulos de vida están disponibles en Comisión Europea, (2010, p. 148).
} 


\section{UNA HERRAMIENTA DE CÁLCULO: LA CALCULADORA SCR}

Centrándonos en los riesgos de mortalidad y de longevidad, los respectivos SCR para una póliza cualquiera vienen determinados en función de:

$$
S C R=f(\text { IRS,Tabla Mortalidad, Activos, Caracteríticas Póliza })
$$

dónde IRS representa la curva IRS (Interest Rate Swap) que se utilice para valorar a precios de mercado, Tabla Mortalidad denota la tabla de mortalidad utilizada, Activos se corresponde con la estructura de activos asociada a la póliza, y Caracteríticas Póliza indica las características de la póliza relevantes para el cálculo del SCR. En concreto, en seguros de vida, edad del asegurado, sexo, temporalidad y estructura de pago de primas.

En el contexto de una determinada compañía, fijar algunos de los componentes de la expresión anterior permitiría simplificar notablemente su aplicación. Así, en primer término y bajo el contexto del modelo estándar, la curva IRS utilizada podría ser la propuesta en Solvencia II, a partir de la cual se obtiene la curva cupón cero que permite valorar las provisiones a precios de mercado tal como se indica en la definición de la valoración Best Estimate. En cuanto a la tabla de mortalidad, esta vendrá prefijada en cada compañía para cada familia de seguros. En este trabajo se supone que la tabla utilizada es la tabla de mortalidad GRM95 (Ministerio de Economía y Competitividad, 2012), la cual para simplificar y no repetir la estrategia para ambos sexos y cada familia de pólizas se admitirá que (i) es la de hombres y (ii) es la misma para todas las pólizas de la compañía. Finalmente, si se presta atención a la estructura de activos, aceptaremos la hipótesis de que permanecen constantes en ambos escenarios. No parece una hipótesis demasiado fuerte ya que activos y pasivos se valoran con la misma curva, tanto para el escenario base como para el escenario después del shock, por lo que la valoración es única.

Así pues, fijadas la tabla de mortalidad, la curva IRS y aceptando una estructura de activos constante, la función que define el SCR resultaría ser:

$$
S C R=f(\text { características de la póliza) }
$$

Conforme a los artículos de las especificaciones técnicas del QIS5, SCR.7.13 y SCR.7.25 $5^{10}$, la aplicación práctica demostrará que para los riesgos de mortalidad y de longevidad, y para los tres tipos de seguro, las pólizas asociadas a cada submódulo de riesgo pueden ser agrupadas y asignadas a una póliza modelo, y adicionalmente demostramos empíricamente que el cálculo predictivo del SCR

${ }^{10}$ Los Artículos SCR.7.13 y SCR.7.25 indican, respectivamente, que cuando se emplean pólizas modelo ("model points") para el cálculo de las provisiones técnicas, y la agrupación de los datos refleje adecuadamente el riesgo de mortalidad/longevidad de la cartera, puede considerarse que cada póliza modelo representa una única póliza a los efectos del sub-módulo. 
asociado será extremadamente sencillo a través de funciones lineales, en las que la cuantía asegurada (importe económico que el asegurado recibe si se produce la contingencia a cubrir) es la variable exógena.

El ejemplo siguiente (Figura 2) ilustra la linealidad de esta última función. La tabla y gráfico asociado recoge los SCR calculados en función de la cantidad asegurada, que corresponden a un individuo de 53 años que contrata un seguro de fallecimiento con prima temporal constante hasta los 65 años.

\section{Figura 2}

Ejemplo de linealidad de la relación entre el SCR y la cuantía asegurada para cada modelo de seguro con hipótesis de estructura de activos constante y condicionando a la estructura de tipos y la tabla de mortalidad

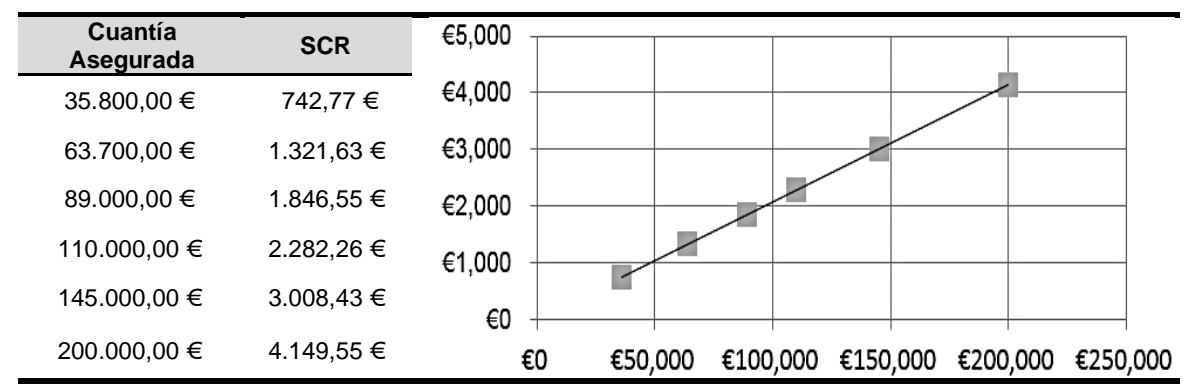

La tabla, y el gráfico asociado, recogen los SCR calculados como función de la cantidad asegurada que corresponden a un individuo de 53 años que contrata un seguro de fallecimiento vitalicio con prima anual constante hasta alcanzar los 65 años de edad.

Fuente: Elaboración propia.

La expresión que liga la provisión predictiva del SCR con las características de la póliza sería por tanto

$$
S C R=a+b^{*} \text { Cuantía Asegurada }
$$

por lo que el problema se reduce a estimar para cada póliza modelo los parámetros $a$ y $b$ que definen la correspondiente recta.

El cálculo analítico de la expresión lineal anterior para el riesgo de mortalidad se recoge en el siguiente epígrafe. Y análogamente se procedería para los distintos casos del riesgo de longevidad recogidos más adelante.

\section{APLICACIÓN}

Vamos a mostrar el funcionamiento de la calculadora SCR a través de algunos ejemplos, centrados en los riesgos de vida: mortalidad y longevidad. Para el riesgo de mortalidad se ha elegido un seguro de fallecimiento con pago de primas constante y periódico; y para el riesgo de longevidad, un seguro de capital diferido y un seguro de renta inmediata. Para llevar a cabo este ejercicio, se formulan las siguientes hipótesis de trabajo: (i) se utilizan las tablas de 
mortalidad GRM95 de varones; (ii) las primas calculadas son primas puras; (iii) el cálculo del Best Estimate se efectúa con la curva de tipos IRS propuesta por el CEIOPS con una prima de iliquidez del 50\%; (iv) el margen de riesgo se considera nulo; y, (v) los activos permanecen constantes en ambos escenarios.

\subsection{Riesgo de mortalidad}

El riesgo de mortalidad se asocia a las obligaciones por seguros y reaseguros en aquellos casos en que una aseguradora garantiza el abono de un solo pago o una serie recurrente de pagos en caso de fallecimiento del tomador del seguro durante la vigencia de la póliza. Aparece cuando el importe a pagar o cuantía asegurada en caso de que se produzca el fallecimiento es superior a las provisiones técnicas dotadas, por lo que el incremento de las tasas de mortalidad generará un incremento en las provisiones técnicas.

En el caso de los contratos que ofrecen prestaciones tanto de fallecimiento como de supervivencia, y tales prestaciones dependen de la vida de la misma persona, no será necesario desvincular las obligaciones. A estos contratos puede aplicárseles plenamente el escenario de mortalidad, teniendo en cuenta el efecto compensatorio proporcionado por la cobertura "natural" entre el componente de prestaciones por fallecimiento y el de prestaciones por supervivencia. En el caso en que después del efecto compensatorio el resultado del escenario sea favorable para el asegurador, se le aplicará un límite de cero.

La carga de capital se calcula como la variación del valor neto de los activos (activos menos pasivos) después de un incremento permanente en las tasas de mortalidad. En nuestro caso los activos van a permanecer constantes en ambos escenarios (antes y después del shock). En línea con el artículo SCR.7 del QIS5, el SCR de mortalidad se calcula a partir de un escenario predefinido:

$$
S C R_{\text {mort }}=\left(\triangle N A V \mid \text { Mortality }_{\text {shock }}\right)
$$

dónde $\triangle N A V$ denota la variación del valor neto de los activos menos los pasivos y Mortality $_{\text {shock }}$ representa un incremento permanente del 15\% en las tasas de mortalidad para cada edad y cada póliza cuando el pago de prestaciones (ya sea pago único o múltiple) depende del riesgo de mortalidad.

A fin de mostrar la simplificación predictiva que supone el uso de la calculadora SCR, mostraremos con un caso particular como se calcularía la predicción del SCR aplicando directamente la metodología propuesta. El ejemplo ilustra cómo se calcula el riesgo de mortalidad para un varón 45 años de edad que paga una prima pura temporal anual constante hasta la edad de los 65 donde la contingencia cubierta es la de fallecimiento con una suma asegurada de $10.000 €$ y con una prima pura de 244,52 € anuales.

Definidas las condiciones de la póliza, el proceso a seguir se describe a través de la siguiente sucesión de tablas hasta obtener finalmente la predicción 
del SCR de la póliza. En la Tabla 1 (donde $\mathrm{q}_{\mathrm{x}}$ denota la probabilidad de que una persona de edad $\mathrm{x}$ fallezca con esa edad $\mathrm{y}_{\mathrm{n}} \mathrm{p}_{\mathrm{x}}$ la de que supere la edad $\mathrm{x}+\mathrm{n}$ ) se representa el valor de mercado de las provisiones técnicas sin que se haya incorporado el shock.

Tabla 1

Valor de mercado de las provisiones técnicas sin incorporar el shock

\begin{tabular}{|c|c|c|c|c|c|c|c|c|}
\hline \multicolumn{9}{|c|}{ Best Estimate: Escenario base } \\
\hline Año & Edad & CF Neto & Prima & $q_{x}$ & ${ }_{n} p_{45}$ & CF esperados & $\begin{array}{c}\text { Curva de } \\
\text { cupón cero }\end{array}$ & $\begin{array}{c}\text { Best } \\
\text { estimate } \\
\text { (MVL) }\end{array}$ \\
\hline 0 & 45 & - & $244,52 €$ & 0,002698 & 1,000000 & $-244,52 €$ & & $-244,52 €$ \\
\hline 1 & 46 & $10.000,00 €$ & $244,52 €$ & 0,002941 & 0,997302 & $-216,87 €$ & $1,4752 \%$ & $-213,72 €$ \\
\hline 2 & 47 & $10.000,00 €$ & $244,52 €$ & 0,003219 & 0,994368 & $-213,81 €$ & $2,0514 \%$ & $-205,30 €$ \\
\hline 3 & 48 & $10.000,00 €$ & $244,52 €$ & 0,003534 & 0,991168 & $-210,35 €$ & $2,4584 \%$ & $-195,57 €$ \\
\hline 4 & 49 & $10.000,00 €$ & $244,52 €$ & 0,003872 & 0,987665 & $-206,48 €$ & $2,7706 \%$ & $-185,09 €$ \\
\hline 69 & 114 & $10.000,00 €$ & $-€$ & 0,390304 & 0,001023 & $6,05 €$ & $3,8981 \%$ & $0,43 €$ \\
\hline 70 & 115 & $10.000,00 €$ & $-€$ & 0,409637 & 0,000624 & $3,99 €$ & $3,9021 \%$ & $0,27 €$ \\
\hline 71 & 116 & $10.000,00 €$ & $-€$ & 0,429498 & 0,000368 & $2,56 €$ & $3,9060 \%$ & $0,17 €$ \\
\hline 72 & 117 & $10.000,00 €$ & $-€$ & 0,449887 & 0,000210 & $1,58 €$ & $3,9099 \%$ &, $10 €$ \\
\hline
\end{tabular}

Fuente: Elaboración propia.

La columna CF esperados recoge la provisión (o cash flow esperado) de la entidad para cada uno de los años, calculada a partir de las tasas de mortalidad, la prima temporal fija $\pi$ y la curva de tipos IRS. En concreto, para un seguro de fallecimiento de vida entera de $C F$ unidades monetarias (10.000 euros en el ejemplo) y pago anual $\pi_{t}$ (donde $\pi_{t}=244,52 €$ para $t \leq 20$ y 0 en otro caso, en el ejemplo) que se contrata por una persona con edad $e$ (45) años, tenemos que el $C F$ esperado en el año 0 vendría dado por:

$$
C F_{\text {Esperados }}(0)=-\pi_{0}{ }_{0} p_{e}=-\pi_{0}
$$

Y para el año $t \geq 1$ :

$$
\begin{aligned}
& C F_{\text {Esperados }}(t)=C F \cdot q_{e+t-1}{ }_{t-1} p_{e}-\pi_{t^{\cdot} t} p_{e}= \\
& =\left(C F \cdot q_{e+t-1}-\pi_{t} \cdot p_{e+t-1}\right) \cdot{ }_{t-1} p_{e}= \\
& =\left(C F \cdot q_{e+t-1}-\pi_{t} \cdot\left(1-q_{e+t-1}\right)\right) \cdot \prod_{j=0}^{t-1}\left(1-q_{e+j}\right)
\end{aligned}
$$

Y para obtener la predicción de las provisiones técnicas basta actualizar a la fecha actual la reserva calculada anteriormente al tipo prefijado por la curva cupón cero, derivada de la curva IRS:

$$
C F_{\text {Esperados }}(t) \cdot\left(1+I R S_{t}\right)^{-t}
$$

donde $I R S_{t}$ es el tipo a aplicar t años adelante. 
La provisión técnica de este producto se obtiene sumando (cambiada de signo) la última columna. En nuestro caso: 672,97 €. En el Anexo I se recoge la tabla completa.

Sin embargo, las especificaciones técnicas del QIS5 exigen recargar la tasa de mortalidad con un incremento del 15\% para calcular la provisión. Al modificarse $q_{x}$ cambian también las probabilidades de supervivencia, de manera que el aumento de la tasa de mortalidad hace que las provisiones aumenten. En la Tabla 2 -elaborada con la misma metodología que la anterior, pero con las $q_{x}$ recargadas- recoge la predicción del nuevo valor de mercado de las provisiones técnicas. En la última columna aparece la variación de las provisiones técnicas actualizadas, cuya suma (cambiada de signo) asciende a 497,08 €. En el Anexo II se recoge la tabla completa.

En este caso, la expresión equivalente para los CF esperados para año $t \geq 1$ resultaría:

$$
C F_{\text {Esperados,stress }}(t)=\left(C F \cdot 1,15 q_{e+t-1}-\pi_{t} \cdot\left(1-1,15 q_{e+t-1}\right)\right) \cdot \prod_{j=0}^{t-1}\left(1-1,15 q_{e+j}\right)
$$

Tabla 2

Valor de mercado de las provisiones técnicas con las $q_{x}$ recargadas

\begin{tabular}{|c|c|c|c|c|c|c|c|c|}
\hline \multicolumn{9}{|c|}{ Best Estimate: Escenario con shock de mortalidad } \\
\hline Año & Edad & CF Neto & Prima & $q_{x}$ & ${ }_{n} p_{45}$ & $\begin{array}{c}\text { CF } \\
\text { esperados }\end{array}$ & $\begin{array}{c}\text { Curva de } \\
\text { cupón } \\
\text { cero }\end{array}$ & $\begin{array}{c}\text { Best } \\
\text { estimate } \\
\text { (MVL) }\end{array}$ \\
\hline 0 & 45 & $€$ & $244,52 €$ & 0,003103 & 1,000000 & $244,52 €$ & & $-244,52 €$ \\
\hline 1 & 46 & $10.000,00 €$ & $244,52 €$ & 0,003383 & 0,996897 & $212,73 €$ & $1,4752 \%$ & $-209,64 €$ \\
\hline 2 & 47 & $10.000,00 €$ & $244,52 €$ & 0,003702 & 0,993525 & $209,21 €$ & $2,0514 \%$ & $-200,89 €$ \\
\hline 3 & 48 & $10.000,00 €$ & $244,52 €$ & 0,004064 & 0,989847 & $205,26 €$ & $2,4584 \%$ & $-190,84 €$ \\
\hline 4 & 49 & $10.000,00 €$ & $244,52 €$ & 0,004452 & 0,985825 & $200,83 €$ & $2,7706 \%$ & $-180,03 €$ \\
\hline \multicolumn{9}{|c|}{$\ldots$} \\
\hline 70 & 115 & $10.000,00 €$ & $-€$ & 0,471083 & 0,000169 & $1,38 €$ & $3,9021 \%$ & $0,09 €$ \\
\hline 71 & 116 & $10.000,00 €$ & $-€$ & 0,493923 & 0,000089 & $0,80 €$ & $3,9060 \%$ & $0,05 €$ \\
\hline 72 & 117 & $10.000,00 €$ & $-€$ & 0,517370 & 0,000045 & $0,44 €$ & $3,9099 \%$ & $0,03 €$ \\
\hline
\end{tabular}

Fuente: Elaboración propia.

Dado que el activo ha permanecido constante, el $\triangle N A V$ se corresponde con la diferencia entre el Best Estimate del Escenario Base y el Best Estimate una vez incorporado el shock, que es igual a 175,89 €.

La estrategia anterior obligaría a recalcular el par de tablas anteriores para cada tipo de póliza o cuando se produjese una modificación en las cuantías aseguradas agregadas de cada tipo de póliza. Como alternativa se podrían calcular los parámetros $a$ y $b$ del modelo lineal que, para cada tipo de póliza, relaciona el SCR con la cuantía asegurada. 
Efectivamente, la relación que liga el SCR con la cuantía asegurada es lineal. Denotando por $\mathrm{PROV}_{0}$ el Best Estimate del Escenario Base, y por $\mathrm{PROV}_{\text {estress }}$ el Best Estimate una vez incorporado el shock, tenemos:

$$
\begin{aligned}
& \operatorname{PROV}_{0}=\sum_{t=0}^{\omega-e} \frac{C F_{\text {Esperados }}(t)}{\left(1+I R S_{t}\right)^{t}} \\
& P R O V_{\text {stress }}=\sum_{t=0}^{\omega-e} \frac{C F_{E_{\text {Esperados,stress }}(t)}}{\left(1+I R S_{t}\right)^{t}}
\end{aligned}
$$

y operando algebraicamente resulta finalmente:

$$
S C R=P R O V_{0}-P R O V_{\text {stress }}=A+B \cdot C F
$$

Con

$$
\begin{gathered}
A=\sum_{t=0}^{\omega-e} \frac{\pi_{t}\left\{\left(1-1,15 q_{e+t-1}\right) \prod_{j=0}^{t-1}\left(1-1,15 q_{e+j}\right)-\left(1-q_{e+t-1}\right) \prod_{j=0}^{t-1}\left(1-q_{e+j}\right)\right\}}{\left(1+I R S_{t}\right)^{t}} \\
B=\sum_{t=0}^{\omega-e} \frac{q_{e+t-1}\left\{\prod_{j=0}^{t-1}\left(1-q_{e+j}\right)-1,15 \prod_{j=0}^{t-1}\left(1-1,15 q_{e+j}\right)\right\}}{\left(1+I R S_{t}\right)^{t}}
\end{gathered}
$$

Dada la complejidad de cálculo de las expresiones de A y B, y teniendo en cuenta que la relación es lineal, puede resultar más sencillo obtener la recta que pasa por dos puntos $\left(C F_{i}, S C R_{i}\right)_{1=1,2}$, como se propone en este trabajo.

Con metodología análoga se deducen las relaciones lineales correspondientes a los otros riesgos estudiados en este artículo.

Fijada la tabla de mortalidad y el sexo, los elementos que caracterizan un seguro de fallecimiento con pago de prima temporal constante son la edad del asegurado y la temporalidad del seguro. Por lo que para calcular el SCR de toda la cartera de una manera rápida y eficiente sería suficiente con obtener los parámetros para todas las posibles combinaciones de edad y temporalidad y adoptar un esquema matricial. En concreto, si para simplificar consideramos exclusivamente los productos más habituales en el mercado dentro de esta familia de seguros y aceptaremos que este tipo de seguro solo se puede contratar con una edad mínima de 20 años y máxima de 65 con una prima temporal pagadera hasta la edad 65, tendríamos que organizando la información en forma matricial, la predicción del SCR de mortalidad correspondiente a la cartera podría calcularse de manera en extremo sencilla. ${ }^{11}$

En concreto, denotando, respectivamente, por $A_{V E}$ y $B_{V E}$ las matrices de

${ }^{11}$ Obviamente estas simplificaciones no son necesarias, más allá de evitar complicaciones en la exposición. 
coordenadas en el origen $a_{e t}$ y de pendientes $b_{e t}$, que dependerán de la edad $e$ del asegurado y de la temporalidad $t$ del seguro, $A_{V E}=\left(a_{e t}\right)$ y $B_{V E}=\left(b_{e t}\right)$ y por $C_{V E}$ a la matriz compuesta por las cuantías $c_{e t}$ que se corresponden con el valor total asegurado de todos los seguros de temporalidad $t$ años con un asegurado de edad $e, C_{V E}=\left(c_{e t}\right)$, se sigue que la predicción del SCR de la cartera se obtiene de manera inmediata mediante la ecuación:

$$
\operatorname{sum}\left(\operatorname{SCR}_{V E}\right)=\operatorname{sum}\left(A_{V E}+B_{V E} \cdot * \cdot C_{V E}\right)
$$

Dónde $S C R_{V E}$ denota la matriz compuesta por los SCR de todos los seguros asociados a una póliza de edad $e$ y temporalidad $t$ años; .** indica que el producto se realiza elemento a elemento; y $\operatorname{sum}(\cdot)$ es un operador que suma todos los elementos de una matriz. Matricialmente la relación anterior quedaría:

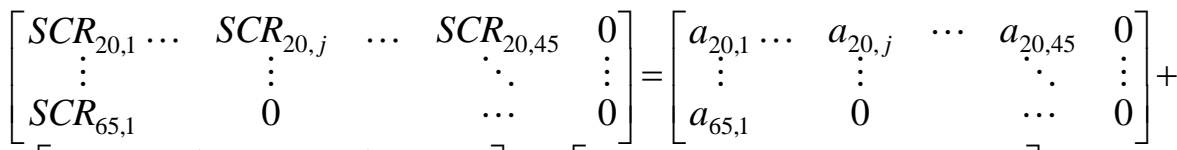

$$
\begin{aligned}
& +\left[\begin{array}{ccccc}
b_{20,1} \cdots & b_{20, j} & \cdots & b_{20,45} & 0 \\
\vdots & \vdots & & \ddots & \vdots \\
b_{65,1} & 0 & & \cdots & 0
\end{array}\right] \cdot * \cdot\left[\begin{array}{ccccc}
c_{20,1} \cdots & c_{20, j} & \cdots & c_{20,45} & 0 \\
\vdots & \vdots & & \ddots & \vdots \\
c_{65,1} & 0 & & \cdots & 0
\end{array}\right]
\end{aligned}
$$

Obsérvese que los triángulos inferiores en estas matrices están todos completos de ceros, ya que la edad máxima de contratación que se ha considerado es de 65 años.

\subsection{Riesgo de longevidad}

Los seguros de longevidad son aquellos que afectan a la existencia del individuo. Concretamente, los seguros de vida o de supervivencia, que son aquellos que cubren el "riesgo" de que el asegurado sobreviva a una determinada fecha o plazo estipulado en el contrato. Solo en este caso la compañía estará obligada a satisfacer la prestación garantizada consistente en la suma o capital asegurado. En este caso, consideraremos dos tipos de seguro de supervivencia de prima única:

- Seguro de capital diferido (Endowment Policy). Cubre el riesgo de longevidad a una determinada fecha, y la prestación toma forma de capital.

- Seguro de renta inmediata. Se garantiza una renta mientras viva el asegurado.

El riesgo de longevidad puede asociarse, en primer lugar, con las rentas. Con la contratación de una renta la compañía garantiza el abono de una serie de pagos temporales hasta el fallecimiento del beneficiario del seguro. Pero también el riesgo afecta a los seguros de capital diferido, en los que existe un abono de pago único en caso de que el tomador del seguro sobreviva durante el periodo de vigencia de la póliza. Por tanto, una disminución en la tasa de 
mortalidad incrementará las provisiones técnicas. Si disminuye la tasa de mortalidad, la probabilidad de vivir aumenta y, por lo tanto, existe una mayor probabilidad de que la aseguradora se vea obligada a pagar durante más años sus rentas o a realizar un mayor porcentaje de pagos únicos.

Este tipo de riesgo es aplicable para las obligaciones por seguros y reaseguros dependientes del riesgo de longevidad, por lo que, o no existe prestación por fallecimiento, o el importe pagadero en caso de fallecimiento es inferior a las provisiones técnicas dotadas y, en consecuencia, la disminución en las tasas de mortalidad dará probablemente lugar a un aumento de éstas.

El capital de solvencia se calculará como la variación en el valor neto de los activos (activos menos pasivos) a consecuencia de una disminución permanente de las tasas de mortalidad. Al igual que ocurre en el caso del riesgo de mortalidad, en los contratos que ofrecen prestaciones tanto de fallecimiento como de supervivencia, y tales prestaciones dependen de la vida de la misma persona, no será necesario desvincular las obligaciones. A estos contratos puede aplicárseles plenamente el escenario de longevidad, teniendo en cuenta el efecto compensatorio proporcionado por la cobertura "natural" entre el componente de prestaciones por fallecimiento y el de prestaciones por supervivencia.

Según las especificaciones técnicas del QIS5, la predicción del capital de solvencia para el riesgo de longevidad se calcula a partir del siguiente escenario de longevidad:

$$
S C R_{\text {long }}=\left(\triangle N A V \mid \text { Longevity }_{\text {shock }}\right)
$$

dónde $\triangle N A V$ denota la variación del valor neto de los activos menos los pasivos y Longevity $_{\text {shock }}$ representa la disminución permanente del $20 \%$ en las probabilidades de mortalidad para cada edad.

Como este tipo de riesgo se asocia básicamente con dos tipos de productos diferentes, se plantean sendos ejemplos.

\section{Seguro de capital diferido}

Un "endowment policy", como se conoce en el mundo asegurador, es un seguro de vida que asegura el pago de un capital si el asegurado vive transcurridos $n$ años. La prima debe servir para pagar la suma asegurada si sobrevive el asegurado. Si el asegurado fallece antes de transcurridos $n$ años no obtiene nada a cambio.

Veamos un breve ejemplo de predicción del SCR en la línea seguida en el apartado anterior. Un varón de 43 años contrata una póliza de seguro de capital diferido, pagando una prima única por la que recibirá una cantidad de 50.000,00 $€$ si llega con vida a los 60 años (con lo que la prima única con la IRS de Solvencia II sería de 27.835,85 €).

Las tablas resumen de los que se deriva la predicción del SCR son como sigue. 
En la Tabla 3 se muestra el escenario base, calculado a precios de mercado. Se observa que la columna de flujos esperados son todos ceros excepto en la edad de los 60 años, que es la única edad en la que puede producirse el pago.

Tabla 3

Escenario base a precios de mercado

\begin{tabular}{|c|c|c|c|c|c|c|c|c|c|}
\hline \multicolumn{10}{|c|}{ Best Estimate: Escenario Base } \\
\hline Año & Edad & CF Neto & $q_{x}$ & ${ }_{n} p_{43}$ & $\begin{array}{c}\text { CF } \\
\text { esperados }\end{array}$ & $\begin{array}{c}\text { Curva de } \\
\text { cupón } \\
\text { cero }\end{array}$ & $\begin{array}{c}\text { Best } \\
\text { estimate } \\
\text { (MVL) }\end{array}$ & $\begin{array}{c}\text { Costes } \\
\text { esperados }\end{array}$ & $\begin{array}{c}\text { MV } \\
\text { de costes } \\
\text { esperados }\end{array}$ \\
\hline 0 & 43 & $-€$ & 0,002299 & 1 & $-€$ & & $-€$ & $250,00 €$ & $250,00 €$ \\
\hline 1 & 44 & $-€$ & 0,002485 & 0,997701 & $-€$ & $1,475 \%$ & $-€$ & $249,43 €$ & $245,80 €$ \\
\hline 2 & 45 & $-€$ & 0,002698 & 0,995221 & $-€$ & $2,051 \%$ & $-€$ & $248,81 €$ & $238,90 €$ \\
\hline 3 & 46 & $-€$ & 0,002941 & 0,992536 & $-€$ & $2,458 \%$ & $-€$ & $248,13 €$ & $230,70 €$ \\
\hline 4 & 47 & $-€$ & 0,003219 & 0,989616 & $-€$ & $2,771 \%$ & $-€$ & $247,40 €$ & $221,78 €$ \\
\hline \multicolumn{10}{|c|}{$\ldots$} \\
\hline 15 & 58 & $-€$ & 0,007962 & 0,935634 & $-€$ & $4,293 \%$ & $-€$ & $233,91 €$ & $124,52 €$ \\
\hline 16 & 59 & $-€$ & 0,008636 & 0,928184 & $-€$ & $4,281 \%$ & $-€$ & $232,05 €$ & $118,66 €$ \\
\hline 17 & 60 & $50.000 €$ & 0,009374 & 0,920168 & $46.008,42 €$ & $4,257 \%$ & $22.650,81 €$ & $-€$ & $-€$ \\
\hline
\end{tabular}

Fuente: Elaboración propia.

La Tabla 4 muestra los flujos valorados a precios de mercado una vez reducidas las tasas de mortalidad en un $20 \%$.

Tabla 4

Flujos a precios de mercado tras reducir las tasas de mortalidad un $20 \%$

\begin{tabular}{|c|c|c|c|c|c|c|c|c|c|}
\hline \multicolumn{10}{|c|}{ Best Estimate: Escenario con reducción de las tasas de mortalidad } \\
\hline Año & Edad & CF Neto & $q_{x}$ & ${ }_{n} p_{43}$ & $\begin{array}{c}\text { CF } \\
\text { esperados }\end{array}$ & $\begin{array}{c}\text { Curva de } \\
\text { cupón } \\
\text { cero }\end{array}$ & $\begin{array}{c}\text { Best } \\
\text { estimate } \\
\text { (MVL) }\end{array}$ & $\begin{array}{c}\text { Costes } \\
\text { esperados }\end{array}$ & $\begin{array}{c}\text { MV } \\
\text { de costes } \\
\text { esperados }\end{array}$ \\
\hline 0 & 43 & $-€$ & 0,001839 & 1 & $-€$ & & $-€$ & $250,00 €$ & $250,00 €$ \\
\hline 1 & 44 & $-€$ & 0,001988 & 0,998161 & $-€$ & $1,475 \%$ & $-€$ & $249,54 €$ & $245,91 €$ \\
\hline 2 & 45 & $-€$ & 0,002159 & 0,996176 & $-€$ & $2,051 \%$ & $-€$ & $249,04 €$ & $239,13 €$ \\
\hline 3 & 46 & $-€$ & 0,002353 & 0,994026 & $-€$ & $2,458 \%$ & $-€$ & $248,51 €$ & $231,04 €$ \\
\hline 4 & 47 & $-€$ & 0,002575 & 0,991687 & $-€$ & $2,771 \%$ & $-€$ & $247,92 €$ & $222,25 €$ \\
\hline \multicolumn{10}{|c|}{$\ldots$} \\
\hline 15 & 58 & $-€$ & 0,006370 & 0,948192 & $-€$ & $4,293 \%$ & $-€$ & $237,05 €$ & $126,19 €$ \\
\hline 16 & 59 & $-€$ & 0,006909 & 0,942152 & $-€$ & $4,281 \%$ & $-€$ & $235,54 €$ & $120,45 €$ \\
\hline 17 & 60 & $50.000 €$ & 0,007500 & 0,935643 & $46.782,16 €$ & $4,257 \%$ & $23.031,74 €$ & $-€$ & $-€$ \\
\hline
\end{tabular}

Fuente: Elaboración propia.

Por hipótesis, los activos permanecen constantes en ambos escenarios. Por tanto, el SCR es igual a la diferencia entre las provisiones valoradas a precio de mercado una vez se ha incorporado el shock y las provisiones, calculadas también a precio de mercado, en el escenario base, que para este ejemplo se concretan en 396,45 €. 
De nuevo los cálculos pueden simplificarse a partir de disponer de los valores de los parámetros $a$ y $b$ para cada modelo de seguro. En este caso el valor de los parámetros depende de la edad del asegurado y del período de diferimiento (o de la edad que el aseguro debería alcanzar para percibir el capital asegurado). En este caso, la información necesaria para los cálculos de la predicción debe representarse de nuevo matricialmente, dado que pueden existir pólizas en las que la edad del asegurado sea la misma pero no coincida la edad de recepción del beneficio. En concreto, denotando, respectivamente, por $\mathrm{A}_{\mathrm{CD}} \mathrm{y}$ $\mathrm{B}_{\mathrm{CD}}$ las matrices de parámetros $\mathrm{a}_{\mathrm{en}} \mathrm{y} \mathrm{b}_{\mathrm{en}}$ donde e indica la edad del asegurado en el momento de la contratación y $\mathrm{n}$ la edad de recepción del beneficio, $\mathrm{A}_{\mathrm{CD}}=\left(\mathrm{a}_{\mathrm{en}}\right)$ y $\mathrm{B}_{\mathrm{CD}}=\left(\mathrm{b}_{\mathrm{en}}\right)$, y por $C_{C D}$ la matriz formada por el agregado de todas las cuantías que se corresponden con el importe total asegurado asociado a todos los seguros con un asegurado de edad $e$ y con edad de recepción del beneficio $n$ años. $C_{C D}=\left(c_{e n}\right)$, se sigue que el SCR de la cartera es

$$
\operatorname{sum}\left(S C R_{C D}\right)=\operatorname{sum}\left(A_{C D}+B_{C D} \cdot * \cdot C_{C D}\right)
$$

donde de nuevo .*. indica que el producto se realiza elemento a elemento. Por ejemplo, estableciendo como hipótesis que la edad mínima de contratación de un seguro de capital diferido es de 20 años y la máxima de 65, matricialmente se expresaría como:

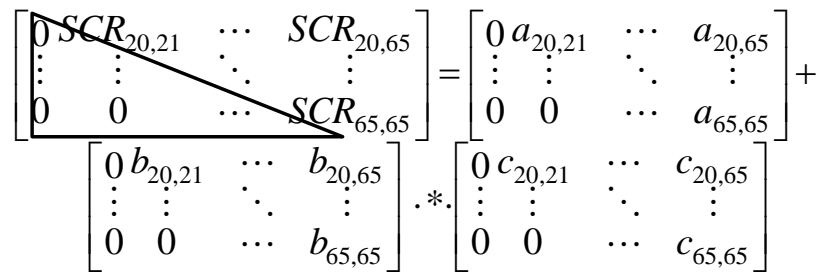

Este caso presenta una peculiaridad. Al definir las columnas como edad de recepción de beneficio, el triángulo inferior de la matriz siempre será 0 , pues no es posible que una persona de edad $e$ reciba la suma asegurada cuando $n<e$. Hay que observar, no obstante, que la representación lineal de este tipo de seguro también se podría haber realizado como en el caso del seguro de fallecimiento. Análogamente, para determinar el SCR de la cartera no hay más que sumar todos los elementos de la matriz $S C R_{C D}$.

Como muestra, en la Figura 3 se presentan gráficamente los valores de los interceptos, coeficientes $a$, y de las pendientes, coeficientes $b$, de las columnas de las matrices $A_{C D}$ y $B_{C D}$ correspondientes a una edad de recepción del beneficio de 60 años, de donde, por ejemplo, se podría calcular directamente el SCR del ejemplo anterior que podría obtenerse fácilmente empleando que $a_{43,60}=15,525$ y que $b_{43,60}=0,0076185(396,45=15,525+0,0076185 \cdot 50000)$ 
Figura 3

Interceptos (gráfico izquierdo) y pendientes (gráfico derecho) en función de la edad para el cálculo del SCR correspondientes a un seguro capital diferido pagadero a la edad de 60 años
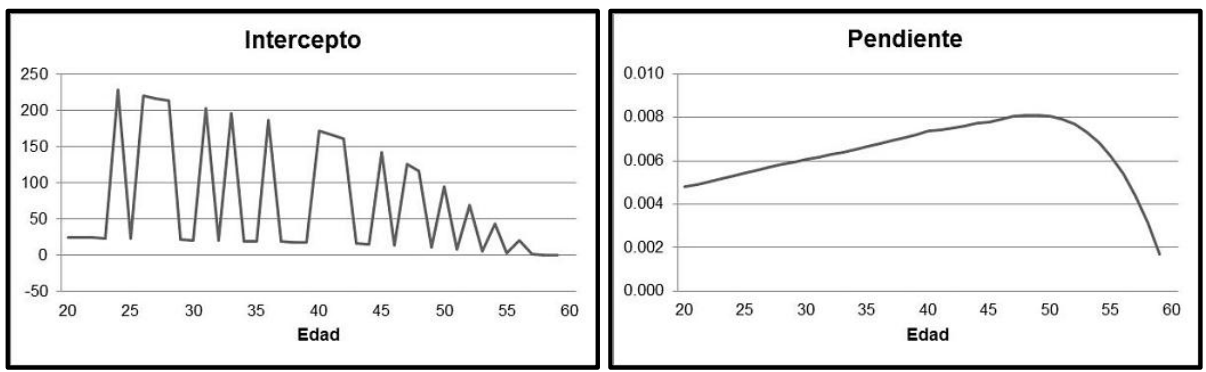

Fuente: Elaboración propia.

\section{Seguros de renta inmediata}

En este tipo de seguro de vida, el asegurado a cambio de una prima única se garantiza una renta inmediata, vitalicia (o temporal) mientras viva.

Al igual de los que en los apartados anteriores para el cálculo de la predicción del SCR desarrollemos, siguiendo la metodología de Solvencia II, un ejemplo. Varón de 60 años que contrata una renta inmediata vitalicia de $6.000 €$, para lo cual ha pagado una prima de 94.986,09 $€$. La renta se comienza a percibir el primer año después del pago.

De las Tablas resumen 5 y 6 se deriva el cálculo predictivo del SCR, tanto para el escenario base como para el escenario de longevidad.

\section{Tabla 5}

Cálculo predictivo del SCR. Escenario base

\begin{tabular}{|c|c|c|c|c|c|c|c|}
\hline \multicolumn{8}{|c|}{ Best Estimate Escenario Base } \\
\hline Año & Edad & CF Neto & $q_{x}$ & ${ }_{n} p_{60}$ & CF Esperado & $\begin{array}{l}\text { Curva de } \\
\text { cupón cero }\end{array}$ & $\begin{array}{c}\text { Best } \\
\text { estimate } \\
\text { (MVL) }\end{array}$ \\
\hline 0 & 60 & $€$ & 0,009374 & 1,000000 & $€$ & & $-€$ \\
\hline 1 & 61 & $6.000,00 €$ & 0,010183 & 0,990626 & $5.943,75 €$ & $1,475 \%$ & $5.857,35 €$ \\
\hline 2 & 62 & $6.000,00 €$ & 0,011067 & 0,980538 & $5.883,23 €$ & $2,051 \%$ & $5.649,09 €$ \\
\hline 3 & 63 & $6.000,00 €$ & 0,011958 & 0,969687 & $5.818,12 €$ & $2,458 \%$ & $5.409,29 €$ \\
\hline 4 & 64 & $6.000,00 €$ & 0,012818 & 0,958091 & $5.748,55 €$ & $2,771 \%$ & $5.153,26 €$ \\
\hline \multicolumn{8}{|c|}{$\cdots$} \\
\hline 58 & 118 & $6.000,00 €$ & 0,470804 & 0,000125 & $0,75 €$ & $3,848 \%$ & $0,08 €$ \\
\hline 59 & 119 & $6.000,00 €$ & 0,492248 & 0,000066 & $0,40 €$ & $3,853 \%$ & $0,04 €$ \\
\hline 60 & 120 & $6.000,00 €$ & 0,514221 & 0,000034 & $0,20 €$ & $3,858 \%$ & $0,02 €$ \\
\hline 61 & 121 & $6.000,00 €$ & 0,536722 & 0,000016 & $0,10 €$ & $3,863 \%$ & $0,01 €$ \\
\hline 62 & 122 & $6.000,00 €$ & 0,559750 & 0,000008 & $0,05 €$ & $3,868 \%$ & $0,00 €$ \\
\hline
\end{tabular}

Fuente: Elaboración propia. 
Para determinar cuáles serían las ecuaciones lineales para este tipo de seguros y así obtener de ellas los SCR de las pólizas modelo y el SCR de una cartera de asegurados, debe tenerse en cuenta una diferencia de esta situación respecto las anteriores. En este caso los parámetros $a$ y $b$ sólo dependen de la edad del asegurado, la prima es única y no existe temporalidad, ya que el seguro se termina cuando el asegurado fallece. Por tanto, en este tipo de pólizas las relaciones se pueden expresar en términos vectoriales.

En concreto, la expresión final de la póliza modelo será:

$$
\overrightarrow{S C R_{R I}}=\vec{a}+\vec{b} * \vec{c}
$$

Donde $\vec{a}$ es el vector fila asociado a las coordenadas en el origen; $\vec{b}$ es el vector fila asociado a la pendiente de la recta; y $\vec{c}$ es el vector columna asociado a las cuantías. Matricialmente:

$$
\left(\begin{array}{lll}
S C R_{20} & \cdots & S C R_{\omega}
\end{array}\right)=\left(\begin{array}{lll}
a_{20} & \cdots & a_{\omega}
\end{array}\right)+\left(\begin{array}{lll}
b_{20} & \cdots & b_{\omega}
\end{array}\right) *\left(\begin{array}{c}
c \\
\vdots \\
c_{w}
\end{array}\right)
$$

Tabla 6

\begin{tabular}{|c|c|c|c|c|c|c|c|}
\hline \multicolumn{8}{|c|}{ Best Estimate Escenario de longevidad } \\
\hline Año & Edad & CF Neto & $q_{x}$ & $n p_{60}$ & CF Esperado & $\begin{array}{l}\text { Curva de } \\
\text { cupón cero }\end{array}$ & $\begin{array}{c}\text { Best } \\
\text { estimate } \\
\text { (MVL) }\end{array}$ \\
\hline 0 & 60 & $€$ & 0,009374 & 1,000000 & $€$ & 0,00 & $€$ \\
\hline 1 & 61 & $6.000,00 €$ & 0,010183 & 0,990626 & $5.955,00 €$ & $1,475 \%$ & $5.868,43 €$ \\
\hline 2 & 62 & $6.000,00 €$ & 0,011067 & 0,980538 & $5.906,49 €$ & $2,051 \%$ & $5.671,42 €$ \\
\hline 3 & 63 & $6.000,00 €$ & 0,011958 & 0,969687 & $5.854,20 €$ & $2,458 \%$ & $5.442,83 €$ \\
\hline 4 & 64 & $6.000,00 €$ & 0,012818 & 0,958091 & $5.798,20 €$ & $2,771 \%$ & $5.197,77 €$ \\
\hline \multicolumn{8}{|c|}{$\cdots$} \\
\hline 58 & 118 & $6.000,00 €$ & 0,470804 & 0,000125 & $5,82 €$ & $3,848 \%$ & $0,65 €$ \\
\hline 59 & 119 & $6.000,00 €$ & 0,492248 & 0,000066 & $3,63 €$ & $3,853 \%$ & $0,39 €$ \\
\hline 60 & 120 & $6.000,00 €$ & 0,514221 & 0,000034 & $2,20 €$ & $3,858 \%$ & $0,23 €$ \\
\hline 61 & 121 & $6.000,00 €$ & 0,536722 & 0,000016 & $1,29 €$ & $3,863 \%$ & $0,13 €$ \\
\hline 62 & 122 & $6.000,00 €$ & 0,559750 & 0,000008 & $0,74 €$ & $3,868 \%$ & $0,07 €$ \\
\hline
\end{tabular}

Cálculo predictivo del SCR. Escenario de longevidad

Fuente: Elaboración propia.

Sumando todos los elementos del vector $S C R_{R I}$ se obtiene el SCR de la cartera.

A modo de ejemplo, la ecuación de la recta que permitiría obtener la predicción del SCR para pólizas similares para un asegurado de 60 años de edad vendría dada por:

$$
S C R_{R V}=-0,00499999999010+0,8687975 *^{*}
$$




\section{CONCLUSIONES}

En términos generales y respecto a Solvencia II, cabe señalar que la incorporación de la nueva Directiva al negocio de los seguros y reaseguros supone entre otras las siguientes consecuencias:

(i) Solvencia II exigirá unos requerimientos de capital acorde con los riesgos que asuma cada entidad. Esto se traducirá en un aumento de capital global. Se trata de una política prudencial para las entidades aseguradoras.

(ii) Los requisitos de solvencia perjudicarán sobre todo al seguro de vida. La necesidad de tener más recursos propios podría encarecer pólizas para mantener los niveles actuales de beneficios de las aseguradoras. En consecuencia, el sector de seguros podría perder atractivo en los mercados de capitales ya que se disminuiría su papel como inversor, tan importante en su actual actividad.

(iii) Si, como contramedida, se adopta la exigencia de mantener políticas de inversión poco agresivas, el rendimiento se vería significativamente reducido.

Respecto la aplicación metodológica desarrollada, ésta ha aportado una visión simplificadora de cómo se puede estimar el capital de solvencia obligatorio (SCR) para los riesgos de mortalidad y longevidad en el negocio de vida. Buena parte de las aseguradoras españolas ya usan técnicas cuantitativas para el cálculo del SCR. En este trabajo se ha propuesto una técnica predictiva muy sencilla, basada en algunas hipótesis bastante realistas, que cuantifica el capital de solvencia obligatorio.

Es destacable que:

(i) El capital de solvencia es una función lineal de la cuantía asegurada, lo que, por una parte, implica que los requerimientos relativos de capital no decrecen con el tamaño de la cartera y, por otra, supone que agrupando el total de pólizas de una cartera según la edad del asegurado y/o la temporalidad del seguro puede definirse una póliza modelo para calcular de forma casi inmediata el capital requerido de solvencia de una determinada cartera y para un riesgo determinado.

Asimismo, disponer de una calculadora para el cálculo predictivo del SCR aporta dos importantes ventajas:

(i) Con la ayuda de la calculadora se reduce significativamente el tiempo de cálculo de las predicciones del SCR correspondiente a cada subriesgo. En concreto, lo que sin la calculadora debe realizarse con la ayuda de los cuadros-plantilla, puede realizarse fácilmente a través de una sencilla regresión.

(ii) Con el uso de la calculadora se disminuye el riesgo operacional y, 
consecuentemente, el SCR global de una entidad de seguros. El riesgo operacional proviene de un desajuste o un fallo en los procesos internos personal y de los sistemas- o de sucesos externos. La simplificación del cálculo a una función lineal reduce las posibilidades de que ocurran fallos humanos, disminuyendo así uno de los componentes que intervienen en el cálculo global del capital de solvencia de una entidad aseguradora.

Finalmente, es preciso destacar que en este trabajo sólo se han tratado dos de los siete subriesgos del módulo de vida: mortalidad y longevidad. Entre los submódulos de vida no analizados, el riesgo de dependencia es el que más similitudes presenta con los estudiados; la dificultad de disponer de tablas de dependencia (Bolancé et al., 2013), sin embargo, ha imposibilitado extender los análisis a este riesgo. En todo caso, quedaría pendiente analizar si para el resto de riesgos (de este módulo y del resto) es aplicable una metodología predictiva similar. Análisis que no es sencillo ya que para algunos riesgos existe todavía una falta de calibración de la cuestión por parte de las autoridades supervisoras.

\section{REFERENCIAS BIBLIOGRÁFICAS}

ABAD, J. y GUTIÉRREZ, C. (2014). "Evaluación de la solvencia bancaria. Un modelo basado en las pruebas de resistencia de la banca española". Estudios de Economía Aplicada, 32(2), pp. 593-616.

ALBARRÁN, I. y ALONSO, P. (2010). Métodos estocásticos de estimación de las provisiones técnicas en el marco de Solvencia II. Madrid: Fundación Mapfre.

ALONSO, P. y ALBARRÁN, I. (2008). Análisis del riesgo en seguros en el marco de Solvencia II: Técnicas estadísticas avanzadas Montecarlo y Bootstrapping. Madrid: Fundación Mapfre.

BOLANCÉ, C.; ALEMANY, R. y GUILLÉN, M. (2013). "Sistema público de dependencia y reducción del coste individual de cuidados a lo largo de la vida". Revista de Economía Aplicada, XXI(61), pp. 97-117.

CAMACHO, A. (2009). Solvencia II: supervisión basada en riesgo de entidades aseguradoras en el marco de la Unión Europea. https://www3.bcu.gub.uy/autoriza/ peiees/jor/2009/iess03j3441009.pdf.

CHRISTIANSEN, M.C. y NIEMEYER, A. (2014). "Fundamental definition of the Solvency Capital Requirement in Solvency II". ASTIN Bulletin: The Journal of the IAA, 44(3), pp. 501-533.

COMISIÓN EUROPEA (2010). Especificaciones técnicas QIS5. Documento de trabajo, Dirección General de Mercado Interior y de Servicios. https://eiopa.europa.eu/ index.php?option=content\&task=view\&id=732.

FERRI, A. (2012). Estructuras de dependencia aplicadas a la gestión de riesgos en Solvencia II. Barcelona: Universitat de Barcelona (Tesis Doctoral).

FRÖHLICH, A. y WENG, A. (2015). "Modelling parameter uncertainty for risk capital calculation". European Actuarial Journal, 5(1), pp. 79-112. 
FRUET-CARDOZO, J.V.; CAÑAS-MADUEÑO, J.A. y MILLÁN DE LA LASTRA, J.R. (2014). “¿Cómo las agencias internacionales de rating califican a España? Criterios para el análisis del Riesgo-País y para determinar la Prima de Riesgo". Estudios de Economía Aplicada, 32(3), pp. 1161-1188.

GARCÍA, S. y VICENS, J. (2006). "Factores condicionantes en la medición del riesgo soberano en los países emergentes". Estudios de Economía Aplicada, 24(1), pp. 245272.

MINISTERIO DE ECONOMIA Y COMPETITIVIDAD (2012). Resolución de 6 de julio de 2012, de la Dirección General de Seguros y Fondos de Pensiones. http://boe.es/boe/ dias/2012/07/21/.

PARLAMENTO EUROPEO Y CONSEJO (2009). Directiva 2009/138/CE, del 25 de noviembre de 2009, sobre el seguro de vida, el acceso a la actividad de seguro y de reaseguro y su ejercicio (Solvencia II). http://eur-lex.europa.eu/JOHtml.do?uri=OJ:L: 2009:335:SOM:EN:HTML.

POZUELO DE GRACIA, E. (2007). "Solvencia II: Capital económico en Aseguradoras". Revista de Economía Financiera, 16(3), pp. 78-110.

ROSADO, B. y ALONSO, J.J. (2015). "Reformas de las pensiones públicas y privadas en España: Persiguiendo la solvencia y la suficiencia". Estudios de Economía Aplicada, 33(3), pp. 859-880.

SANDSTRÖM, A. (2006). Solvency: Models, Assessment and Regulation. New York: Chapman \& Hall/CRC. 


\section{Anexo I}

\begin{tabular}{|c|c|c|c|c|c|c|c|c|}
\hline \multicolumn{9}{|c|}{ Best Estimate Escenario Base } \\
\hline Year & Age & Net CF & Premium & $\begin{array}{l}\text { 1-year } \\
\text { Mortality } \\
\text { rates }\end{array}$ & $\begin{array}{l}\text { n-year } \\
\text { survivor } \\
\text { rates }\end{array}$ & Expected CF & $\begin{array}{l}\text { Zero } \\
\text { Curve }\end{array}$ & $\begin{array}{c}\text { Best } \\
\text { estimate } \\
\text { (MVL) }\end{array}$ \\
\hline 0 & 45 & $-€$ & $244,52 €$ & 0,002698 & 1,000000 & $-244,52 €$ & & $-244,52 €$ \\
\hline 1 & 46 & $10.000,00 €$ & $244,52 €$ & 0,002941 & 0,997302 & $-216,87 €$ & $1,4752 \%$ & $-213,72 €$ \\
\hline 2 & 47 & $10.000,00 €$ & $244,52 €$ & 0,003219 & 0,994368 & $-213,81 €$ & $2,0514 \%$ & $-205,30 €$ \\
\hline 3 & 48 & $10.000,00 €$ & $244,52 €$ & 0,003534 & 0,991168 & $-210,35 €$ & $2,4584 \%$ & $-195,57 €$ \\
\hline 4 & 49 & $10.000,00 €$ & $244,52 €$ & 0,003872 & 0,987665 & $-206,48 €$ & $2,7706 \%$ & $-185,09 €$ \\
\hline 5 & 50 & $10.000,00 €$ & $244,52 €$ & 0,004218 & 0,983841 & $-202,33 €$ & $3,0217 \%$ & $-174,35 €$ \\
\hline 6 & 51 & $10.000,00 €$ & $244,52 €$ & 0,004578 & 0,979692 & $-198,06 €$ & $3,2350 \%$ & $-163,62 €$ \\
\hline 7 & 52 & $10.000,00 €$ & $244,52 €$ & 0,004958 & 0,975207 & $-193,61 €$ & $3,4225 \%$ & $-152,97 €$ \\
\hline 8 & 53 & $10.000,00 €$ & $244,52 €$ & 0,005363 & 0,970372 & $-188,93 €$ & $3,5895 \%$ & $-142,48 €$ \\
\hline 9 & 54 & $10.000,00 €$ & $244,52 €$ & 0,005799 & 0,965169 & $-183,96 €$ & $3,7384 \%$ & $-132,21 €$ \\
\hline 10 & 55 & $10.000,00 €$ & $244,52 €$ & 0,006271 & 0,959572 & $-178,67 €$ & $3,8703 \%$ & $-122,22 €$ \\
\hline 11 & 56 & $10.000,00 €$ & $244,52 €$ & 0,006785 & 0,953555 & $-172,99 €$ & $3,9859 \%$ & $-112,54 €$ \\
\hline 12 & 57 & $10.000,00 €$ & $244,52 €$ & 0,007347 & 0,947085 & $-166,88 €$ & $4,0855 \%$ & $-103,21 €$ \\
\hline 13 & 58 & $10.000,00 €$ & $244,52 €$ & 0,007962 & 0,940127 & $-160,30 €$ & $4,1694 \%$ & $-94,25 €$ \\
\hline 14 & 59 & $10.000,00 €$ & $244,52 €$ & 0,008636 & 0,932641 & $-153,19 €$ & $4,2381 \%$ & $-85,68 €$ \\
\hline 15 & 60 & $10.000,00 €$ & $244,52 €$ & 0,009374 & 0,924587 & $-145,53 €$ & $4,2926 \%$ & $-77,48 €$ \\
\hline 16 & 61 & $10.000,00 €$ & $244,52 €$ & 0,010183 & 0,915919 & $-137,28 €$ & $4,2807 \%$ & $-70,20 €$ \\
\hline 17 & 62 & $10.000,00 €$ & $244,52 €$ & 0,011067 & 0,906593 & $-128,41 €$ & $4,2565 \%$ & $-63,22 €$ \\
\hline 18 & 63 & $10.000,00 €$ & $244,52 €$ & 0,011958 & 0,896560 & $-118,89 €$ & $4,2211 \%$ & $-56,49 €$ \\
\hline 19 & 64 & $10.000,00 €$ & $244,52 €$ & 0,012818 & 0,885839 & $-109,39 €$ & $4,1756 \%$ & $-50,28 €$ \\
\hline 20 & 65 & $10.000,00 €$ & $244,52 €$ & 0,013697 & 0,874484 & $-100,28 €$ & $4,1213 \%$ & $-44,71 €$ \\
\hline 21 & 66 & $10.000,00 €$ & $-€$ & 0,014640 & 0,862506 & $119,78 €$ & $4,1124 \%$ & $51,38 €$ \\
\hline 22 & 67 & $10.000,00 €$ & $-€$ & 0,015691 & 0,849879 & $126,27 €$ & $4,0972 \%$ & $52,20 €$ \\
\hline 23 & 68 & $10.000,00 €$ & $-€$ & 0,016891 & 0,836544 & $133,36 €$ & $4,0769 \%$ & $53,19 €$ \\
\hline 24 & 69 & $10.000,00 €$ & $-€$ & 0,018278 & 0,822413 & $141,30 €$ & $4,0528 \%$ & $54,46 €$ \\
\hline 25 & 70 & $10.000,00 €$ & $-€$ & 0,019886 & 0,807382 & $150,32 €$ & $4,0258 \%$ & $56,04 €$ \\
\hline 26 & 71 & $10.000,00 €$ & $-€$ & 0,021750 & 0,791326 & $160,56 €$ & $3,9970 \%$ & $57,95 €$ \\
\hline 27 & 72 & $10.000,00 €$ & $-€$ & 0,023889 & 0,774115 & $172,11 €$ & $3,9669 \%$ & $60,21 €$ \\
\hline 28 & 73 & $10.000,00 €$ & $-€$ & 0,026286 & 0,755622 & $184,93 €$ & $3,9362 \%$ & $62,74 €$ \\
\hline 29 & 74 & $10.000,00 €$ & $-€$ & 0,028914 & 0,735759 & $198,63 €$ & $3,9053 \%$ & $65,39 €$ \\
\hline 30 & 75 & $10.000,00 €$ & $-€$ & 0,031746 & 0,714486 & $212,74 €$ & $3,8745 \%$ & $68,01 €$ \\
\hline 31 & 76 & $10.000,00 €$ & $-€$ & 0,034759 & 0,691804 & $226,82 €$ & $3,8487 \%$ & $70,35 €$ \\
\hline 32 & 77 & $10.000,00 €$ & $-€$ & 0,037928 & 0,667757 & $240,46 €$ & $3,8281 \%$ & $72,27 €$ \\
\hline 33 & 78 & $10.000,00 €$ & $-€$ & 0,041232 & 0,642431 & $253,27 €$ & $3,8120 \%$ & $73,69 €$ \\
\hline 34 & 79 & $10.000,00 €$ & $-€$ & 0,044648 & 0,615942 & $264,88 €$ & $3,7995 \%$ & $74,54 €$ \\
\hline 35 & 80 & $10.000,00 €$ & $-€$ & 0,048159 & 0,588441 & $275,01 €$ & $3,7901 \%$ & $74,80 €$ \\
\hline 36 & 81 & $10.000,00 €$ & $-€$ & 0,051743 & 0,560103 & $283,39 €$ & $3,7833 \%$ & $74,44 €$ \\
\hline 37 & 82 & $10.000,00 €$ & $-€$ & 0,055383 & 0,531121 & $289,81 €$ & $3,7787 \%$ & $73,47 €$ \\
\hline 38 & 83 & $10.000,00 €$ & $-€$ & 0,059062 & 0,501706 & $294,15 €$ & $3,7758 \%$ & $71,93 €$ \\
\hline 39 & 84 & $10.000,00 €$ & $-€$ & 0,062764 & 0,472074 & $296,32 €$ & $3,7744 \%$ & $69,86 €$ \\
\hline 40 & 85 & $10.000,00 €$ & $-€$ & 0,066473 & 0,442445 & $296,29 €$ & $3,7743 \%$ & $67,32 €$ \\
\hline 41 & 86 & $10.000,00 €$ & $-€$ & 0,070175 & 0,413034 & $294,11 €$ & $3,7752 \%$ & $64,37 €$ \\
\hline 42 & 87 & $10.000,00 €$ & $-€$ & 0,073892 & 0,384049 & $289,85 €$ & $3,7770 \%$ & $61,08 €$ \\
\hline 43 & 88 & $10.000,00 €$ & $-€$ & 0,077791 & 0,355671 & $283,78 €$ & $3,7795 \%$ & $57,57 €$ \\
\hline 44 & 89 & $10.000,00 €$ & $-€$ & 0,082060 & 0,328003 & $276,68 €$ & $3,7825 \%$ & $54,01 €$ \\
\hline 45 & 90 & $10.000,00 €$ & $-€$ & 0,086880 & 0,301087 & $269,16 €$ & $3,7861 \%$ & $50,55 €$ \\
\hline 46 & 91 & $10.000,00 €$ & $-€$ & 0,092417 & 0,274929 & $261,58 €$ & $3,7900 \%$ & $47,26 €$ \\
\hline 47 & 92 & $10.000,00 €$ & $-€$ & 0,098826 & 0,249521 & $254,08 €$ & $3,7943 \%$ & $44,14 €$ \\
\hline 48 & 93 & $10.000,00 €$ & $-€$ & 0,106254 & 0,224862 & $246,59 €$ & $3,7988 \%$ & $41,19 €$ \\
\hline 49 & 94 & $10.000,00 €$ & $-€$ & 0,114501 & 0,200969 & $238,93 €$ & $3,8035 \%$ & $38,36 €$ \\
\hline 50 & 95 & $10.000,00 €$ & $-€$ & 0,123277 & 0,177958 & $230,11 €$ & $3,8083 \%$ & $35,51 €$ \\
\hline 51 & 96 & $10.000,00 €$ & $-€$ & 0,132579 & 0,156020 & $219,38 €$ & $3,8133 \%$ & $32,53 €$ \\
\hline 52 & 97 & $10.000,00 €$ & $-€$ & 0,142410 & 0,135335 & $206,85 €$ & $3,8183 \%$ & $29,47 €$ \\
\hline
\end{tabular}




\begin{tabular}{|c|c|c|c|c|c|c|c|c|}
\hline \multicolumn{9}{|c|}{ Best Estimate Escenario Base (Continue) } \\
\hline Year & Age & Net CF & Premium & $\begin{array}{l}\text { 1-year } \\
\text { Mortality } \\
\text { rates }\end{array}$ & $\begin{array}{l}\text { n-year } \\
\text { survivor } \\
\text { rates }\end{array}$ & Expected CF & $\begin{array}{l}\text { Zero } \\
\text { Curve }\end{array}$ & $\begin{array}{c}\text { Best } \\
\text { estimate } \\
\text { (MVL) }\end{array}$ \\
\hline 53 & 98 & $10.000,00 €$ & $-€$ & 0,152769 & 0,116062 & $192,73 €$ & $3,8233 \%$ & $26,38 €$ \\
\hline 54 & 99 & $10.000,00 €$ & $-€$ & 0,163656 & 0,098331 & $177,31 €$ & $3,8284 \%$ & $23,32 €$ \\
\hline 55 & 100 & $10.000,00 €$ & $-€$ & 0,175070 & 0,082239 & $160,92 €$ & $3,8334 \%$ & $20,33 €$ \\
\hline 56 & 101 & $10.000,00 €$ & $-€$ & 0,187013 & 0,067841 & $143,98 €$ & $3,8385 \%$ & $17,47 €$ \\
\hline 57 & 102 & $10.000,00 €$ & $-€$ & 0,199483 & 0,055154 & $126,87 €$ & $3,8435 \%$ & $14,78 €$ \\
\hline 58 & 103 & $10.000,00 €$ & $-€$ & 0,212481 & 0,044152 & $110,02 €$ & $3,8484 \%$ & $12,31 €$ \\
\hline 59 & 104 & $10.000,00 €$ & $-€$ & 0,226008 & 0,034770 & $93,81 €$ & $3,8533 \%$ & $10,08 €$ \\
\hline 60 & 105 & $10.000,00 €$ & $-€$ & 0,240062 & 0,026912 & $78,58 €$ & $3,8581 \%$ & $8,11 €$ \\
\hline 61 & 106 & $10.000,00 €$ & $-€$ & 0,254644 & 0,020451 & $64,61 €$ & $3,8629 \%$ & $6,40 €$ \\
\hline 62 & 107 & $10.000,00 €$ & $-€$ & 0,269753 & 0,015244 & $52,08 €$ & $3,8676 \%$ & $4,95 €$ \\
\hline 63 & 108 & $10.000,00 €$ & $-€$ & 0,285391 & 0,011132 & $41,12 €$ & $3,8722 \%$ & $3,75 €$ \\
\hline 64 & 109 & $10.000,00 €$ & $-€$ & 0,301557 & 0,007955 & $31,77 €$ & $3,8767 \%$ & $2,79 €$ \\
\hline 65 & 110 & $10.000,00 €$ & $-€$ & 0,318250 & 0,005556 & $23,99 €$ & $3,8812 \%$ & $2,02 €$ \\
\hline 66 & 111 & $10.000,00 €$ & $-€$ & 0,335472 & 0,003788 & $17,68 €$ & $3,8855 \%$ & $1,43 €$ \\
\hline 67 & 112 & $10.000,00 €$ & $-€$ & 0,353221 & 0,002517 & $12,71 €$ & $3,8898 \%$ & $0,99 €$ \\
\hline 68 & 113 & $10.000,00 €$ & $-€$ & 0,371499 & 0,001628 & $8,89 €$ & $3,8940 \%$ & $0,66 €$ \\
\hline 69 & 114 & $10.000,00 €$ & $-€$ & 0,390304 & 0,001023 & $6,05 €$ & $3,8981 \%$ & $0,43 €$ \\
\hline 70 & 115 & $10.000,00 €$ & $-€$ & 0,409637 & 0,000624 & $3,99 €$ & $3,9021 \%$ & $0,27 €$ \\
\hline 71 & 116 & $10.000,00 €$ & $-€$ & 0,429498 & 0,000368 & $2,56 €$ & $3,9060 \%$ & $0,17 €$ \\
\hline 72 & 117 & $10.000,00 €$ & $-€$ & 0,449887 & 0,000210 & $1,58 €$ & $3,9099 \%$ & $0,10 €$ \\
\hline 73 & 118 & $10.000,00 €$ & $-€$ & 0,470804 & 0,000116 & $0,95 €$ & $3,9136 \%$ & $0,06 €$ \\
\hline 74 & 119 & $10.000,00 €$ & $-€$ & 0,492248 & 0,000061 & $0,54 €$ & $3,9173 \%$ & $0,03 €$ \\
\hline 75 & 120 & $10.000,00 €$ & $-€$ & 0,514221 & 0,000031 & $0,30 €$ & $3,9209 \%$ & $0,02 €$ \\
\hline 76 & 121 & $10.000,00 €$ & $-€$ & 0,536722 & 0,000015 & $0,16 €$ & $3,9244 \%$ & $0,01 €$ \\
\hline 77 & 122 & $10.000,00 €$ & $-€$ & 0,559750 & 0,000007 & $0,08 €$ & $3,9279 \%$ & $0,00 €$ \\
\hline 78 & 123 & $10.000,00 €$ & $-€$ & 0,583306 & 0,000003 & $0,04 €$ & $3,9312 \%$ & $0,00 €$ \\
\hline 79 & 124 & $10.000,00 €$ & $-€$ & 0,607391 & 0,000001 & $0,02 €$ & $3,9345 \%$ & $0,00 €$ \\
\hline 80 & 125 & $10.000,00 €$ & $-€$ & 0,632003 & 0,000001 & $0,01 €$ & $3,9377 \%$ & $0,00 €$ \\
\hline 81 & 126 & $10.000,00 €$ & $-€$ & 1,000000 & 0,000000 & $0,00 €$ & $3,9409 \%$ & $0,00 €$ \\
\hline
\end{tabular}

Fuente: Elaboración propia. 


\section{Anexo II}

\begin{tabular}{|c|c|c|c|c|c|c|c|c|}
\hline \multicolumn{9}{|c|}{ Best estimate escenario de mortalidad } \\
\hline Year & Age & Net CF & Premium & $\begin{array}{c}\text { 1-year } \\
\text { Mortality } \\
\text { rates }\end{array}$ & $\begin{array}{l}\text { n-year } \\
\text { survivor } \\
\text { rates }\end{array}$ & Expected CF & $\begin{array}{l}\text { Zero } \\
\text { Curve }\end{array}$ & $\begin{array}{c}\text { Best } \\
\text { estimate } \\
\text { (MVL) }\end{array}$ \\
\hline 0 & 45 & $-€$ & $244,52 €$ & 0,003103 & 1,000000 & $-244,52 €$ & & $-244,52 €$ \\
\hline 1 & 46 & $10.000,00 €$ & $244,52 €$ & 0,003383 & 0,996897 & $-212,73 €$ & $1,4752 \%$ & $-209,64 €$ \\
\hline 2 & 47 & $10.000,00 €$ & $244,52 €$ & 0,003702 & 0,993525 & $-209,21 €$ & $2,0514 \%$ & $-200,89 €$ \\
\hline 3 & 48 & $10.000,00 €$ & $244,52 €$ & 0,004064 & 0,989847 & $-205,26 €$ & $2,4584 \%$ & $-190,84 €$ \\
\hline 4 & 49 & $10.000,00 €$ & $244,52 €$ & 0,004452 & 0,985825 & $-200,83 €$ & $2,7706 \%$ & $-180,03 €$ \\
\hline 5 & 50 & $10.000,00 €$ & $244,52 €$ & 0,004850 & 0,981435 & $-196,09 €$ & $3,0217 \%$ & $-168,97 €$ \\
\hline 6 & 51 & $10.000,00 €$ & $244,52 €$ & 0,005264 & 0,976675 & $-191,21 €$ & $3,2350 \%$ & $-157,96 €$ \\
\hline 7 & 52 & $10.000,00 €$ & $244,52 €$ & 0,005701 & 0,971534 & $-186,14 €$ & $3,4225 \%$ & $-147,07 €$ \\
\hline 8 & 53 & $10.000,00 €$ & $244,52 €$ & 0,006167 & 0,965995 & $-180,81 €$ & $3,5895 \%$ & $-136,37 €$ \\
\hline 9 & 54 & $10.000,00 €$ & $244,52 €$ & 0,006668 & 0,960037 & $-175,17 €$ & $3,7384 \%$ & $-125,90 €$ \\
\hline 10 & 55 & $10.000,00 €$ & $244,52 €$ & 0,007211 & 0,953636 & $-169,16 €$ & $3,8703 \%$ & $-115,72 €$ \\
\hline 11 & 56 & $10.000,00 €$ & $244,52 €$ & 0,007803 & 0,946759 & $-162,73 €$ & $3,9859 \%$ & $-105,86 €$ \\
\hline 12 & 57 & $10.000,00 €$ & $244,52 €$ & 0,008449 & 0,939371 & $-155,82 €$ & $4,0855 \%$ & $-96,37 €$ \\
\hline 13 & 58 & $10.000,00 €$ & $244,52 €$ & 0,009156 & 0,931434 & $-148,38 €$ & $4,1694 \%$ & $-87,25 €$ \\
\hline 14 & 59 & $10.000,00 €$ & $244,52 €$ & 0,009932 & 0,922906 & $-140,38 €$ & $4,2381 \%$ & $-78,51 €$ \\
\hline 15 & 60 & $10.000,00 €$ & $244,52 €$ & 0,010781 & 0,913740 & $-131,77 €$ & $4,2926 \%$ & $-70,15 €$ \\
\hline 16 & 61 & $10.000,00 €$ & $244,52 €$ & 0,011710 & 0,903889 & $-122,51 €$ & $4,2807 \%$ & $-62,65 €$ \\
\hline 17 & 62 & $10.000,00 €$ & $244,52 €$ & 0,012727 & 0,893304 & $-112,58 €$ & $4,2565 \%$ & $-55,43 €$ \\
\hline 18 & 63 & $10.000,00 €$ & $244,52 €$ & 0,013752 & 0,881936 & $-101,96 €$ & $4,2211 \%$ & $-48,44 €$ \\
\hline 19 & 64 & $10.000,00 €$ & $244,52 €$ & 0,014741 & 0,869808 & $-91,40 €$ & $4,1756 \%$ & $-42,02 €$ \\
\hline 20 & 65 & $10.000,00 €$ & $244,52 €$ & 0,015751 & 0,856986 & $-81,33 €$ & $4,1213 \%$ & $-36,26 €$ \\
\hline 21 & 66 & $10.000,00 €$ & $-€$ & 0,016836 & 0,843487 & $134,99 €$ & $4,1124 \%$ & $57,91 €$ \\
\hline 22 & 67 & $10.000,00 €$ & $-€$ & 0,018045 & 0,829286 & $142,01 €$ & $4,0972 \%$ & $58,70 €$ \\
\hline 23 & 68 & $10.000,00 €$ & $-€$ & 0,019425 & 0,814322 & $149,64 €$ & $4,0769 \%$ & $59,69 €$ \\
\hline 24 & 69 & $10.000,00 €$ & $-€$ & 0,021019 & 0,798504 & $158,18 €$ & $4,0528 \%$ & $60,96 €$ \\
\hline 25 & 70 & $10.000,00 €$ & $-€$ & 0,022869 & 0,781720 & $167,84 €$ & $4,0258 \%$ & $62,57 €$ \\
\hline 26 & 71 & $10.000,00 €$ & $-€$ & 0,025012 & 0,763843 & $178,77 €$ & $3,9970 \%$ & $64,53 €$ \\
\hline 27 & 72 & $10.000,00 €$ & $-€$ & 0,027473 & 0,744737 & $191,05 €$ & $3,9669 \%$ & $66,83 €$ \\
\hline 28 & 73 & $10.000,00 €$ & $-€$ & 0,030229 & 0,724278 & $204,60 €$ & $3,9362 \%$ & $69,41 €$ \\
\hline 29 & 74 & $10.000,00 €$ & $-€$ & 0,033251 & 0,702383 & $218,94 €$ & $3,9053 \%$ & $72,08 €$ \\
\hline 30 & 75 & $10.000,00 €$ & $-€$ & 0,036508 & 0,679028 & $233,55 €$ & $3,8745 \%$ & $74,66 €$ \\
\hline 31 & 76 & $10.000,00 €$ & $-€$ & 0,039973 & 0,654238 & $247,90 €$ & $3,8487 \%$ & $76,89 €$ \\
\hline 32 & 77 & $10.000,00 €$ & $-€$ & 0,043617 & 0,628087 & $261,52 €$ & $3,8281 \%$ & $78,60 €$ \\
\hline 33 & 78 & $10.000,00 €$ & $-€$ & 0,047416 & 0,600691 & $273,95 €$ & $3,8120 \%$ & $79,71 €$ \\
\hline 34 & 79 & $10.000,00 €$ & $-€$ & 0,051346 & 0,572209 & $284,83 €$ & $3,7995 \%$ & $80,16 €$ \\
\hline 35 & 80 & $10.000,00 €$ & $-€$ & 0,055383 & 0,542828 & $293,81 €$ & $3,7901 \%$ & $79,91 €$ \\
\hline 36 & 81 & $10.000,00 €$ & $-€$ & 0,059504 & 0,512765 & $300,63 €$ & $3,7833 \%$ & $78,97 €$ \\
\hline 37 & 82 & $10.000,00 €$ & $-€$ & 0,063691 & 0,482253 & $305,12 €$ & $3,7787 \%$ & $77,35 €$ \\
\hline 38 & 83 & $10.000,00 €$ & $-€$ & 0,067922 & 0,451538 & $307,15 €$ & $3,7758 \%$ & $75,11 €$ \\
\hline 39 & 84 & $10.000,00 €$ & $-€$ & 0,072179 & 0,420869 & $306,69 €$ & $3,7744 \%$ & $72,31 €$ \\
\hline 40 & 85 & $10.000,00 €$ & $-€$ & 0,076444 & 0,390491 & $303,78 €$ & $3,7743 \%$ & $69,02 €$ \\
\hline 41 & 86 & $10.000,00 €$ & $-€$ & 0,080701 & 0,360640 & $298,51 €$ & $3,7752 \%$ & $65,33 €$ \\
\hline 42 & 87 & $10.000,00 €$ & $-€$ & 0,084976 & 0,331536 & $291,04 €$ & $3,7770 \%$ & $61,34 €$ \\
\hline 43 & 88 & $10.000,00 €$ & $-€$ & 0,089459 & 0,303363 & $281,73 €$ & $3,7795 \%$ & $57,15 €$ \\
\hline 44 & 89 & $10.000,00 €$ & $-€$ & 0,094369 & 0,276225 & $271,39 €$ & $3,7825 \%$ & $52,98 €$ \\
\hline 45 & 90 & $10.000,00 €$ & $-€$ & 0,099912 & 0,250158 & $260,67 €$ & $3,7861 \%$ & $48,96 €$ \\
\hline 46 & 91 & $10.000,00 €$ & $-€$ & 0,106279 & 0,225164 & $249,94 €$ & $3,7900 \%$ & $45,15 €$ \\
\hline 47 & 92 & $10.000,00 €$ & $-€$ & 0,113650 & 0,201234 & $239,30 €$ & $3,7943 \%$ & $41,57 €$ \\
\hline 48 & 93 & $10.000,00 €$ & $-€$ & 0,122192 & 0,178363 & $228,70 €$ & $3,7988 \%$ & $38,20 €$ \\
\hline 49 & 94 & $10.000,00 €$ & $-€$ & 0,131677 & 0,156569 & $217,95 €$ & $3,8035 \%$ & $34,99 €$ \\
\hline 50 & 95 & $10.000,00 €$ & $-€$ & 0,141768 & 0,135952 & $206,16 €$ & $3,8083 \%$ & $31,81 €$ \\
\hline 51 & 96 & $10.000,00 €$ & $-€$ & 0,152466 & 0,116679 & $192,74 €$ & $3,8133 \%$ & $28,58 €$ \\
\hline 52 & 97 & $10.000,00 €$ & $-€$ & 0,163772 & 0,098889 & $177,90 €$ & $3,8183 \%$ & $25,35 €$ \\
\hline 53 & 98 & $10.000,00 €$ & $-€$ & 0,175684 & 0,082694 & $161,95 €$ & $3,8233 \%$ & $22,17 €$ \\
\hline
\end{tabular}




\begin{tabular}{|c|c|c|c|c|c|c|c|c|}
\hline \multicolumn{9}{|c|}{ Best estimate escenario de mortalidad (Continue) } \\
\hline Year & Age & Net CF & Premium & $\begin{array}{c}\text { 1-year } \\
\text { Mortality } \\
\text { rates }\end{array}$ & $\begin{array}{l}\text { n-year } \\
\text { survivor } \\
\text { rates }\end{array}$ & Expected CF & $\begin{array}{l}\text { Zero } \\
\text { Curve }\end{array}$ & $\begin{array}{c}\text { Best } \\
\text { estimate } \\
\text { (MVL) }\end{array}$ \\
\hline 54 & 99 & $10.000,00 €$ & $-€$ & 0,188204 & 0,068166 & $145,28 €$ & $3,8284 \%$ & $19,10 €$ \\
\hline 55 & 100 & $10.000,00 €$ & $-€$ & 0,201331 & 0,055337 & $128,29 €$ & $3,8334 \%$ & $16,21 €$ \\
\hline 56 & 101 & $10.000,00 €$ & $-€$ & 0,215065 & 0,044196 & $111,41 €$ & $3,8385 \%$ & $13,52 €$ \\
\hline 57 & 102 & $10.000,00 €$ & $-€$ & 0,229405 & 0,034691 & $95,05 €$ & $3,8435 \%$ & $11,07 €$ \\
\hline 58 & 103 & $10.000,00 €$ & $-€$ & 0,244353 & 0,026733 & $79,58 €$ & $3,8484 \%$ & $8,90 €$ \\
\hline 59 & 104 & $10.000,00 €$ & $-€$ & 0,259909 & 0,020200 & $65,32 €$ & $3,8533 \%$ & $7,02 €$ \\
\hline 60 & 105 & $10.000,00 €$ & $-€$ & 0,276071 & 0,014950 & $52,50 €$ & $3,8581 \%$ & $5,42 €$ \\
\hline 61 & 106 & $10.000,00 €$ & $-€$ & 0,292840 & 0,010823 & $41,27 €$ & $3,8629 \%$ & $4,09 €$ \\
\hline 62 & 107 & $10.000,00 €$ & $-€$ & 0,310216 & 0,007653 & $31,69 €$ & $3,8676 \%$ & $3,01 €$ \\
\hline 63 & 108 & $10.000,00 €$ & $-€$ & 0,328200 & 0,005279 & $23,74 €$ & $3,8722 \%$ & $2,17 €$ \\
\hline 64 & 109 & $10.000,00 €$ & $-€$ & 0,346790 & 0,003547 & $17,33 €$ & $3,8767 \%$ & $1,52 €$ \\
\hline 65 & 110 & $10.000,00 €$ & $-€$ & 0,365988 & 0,002317 & $12,30 €$ & $3,8812 \%$ & $1,04 €$ \\
\hline 66 & 111 & $10.000,00 €$ & $-€$ & 0,385793 & 0,001469 & $8,48 €$ & $3,8855 \%$ & $0,69 €$ \\
\hline 67 & 112 & $10.000,00 €$ & $-€$ & 0,406204 & 0,000902 & $5,67 €$ & $3,8898 \%$ & $0,44 €$ \\
\hline 68 & 113 & $10.000,00 €$ & $-€$ & 0,427223 & 0,000536 & $3,66 €$ & $3,8940 \%$ & $0,27 €$ \\
\hline 69 & 114 & $10.000,00 €$ & $-€$ & 0,448849 & 0,000307 & $2,29 €$ & $3,8981 \%$ & $0,16 €$ \\
\hline 70 & 115 & $10.000,00 €$ & $-€$ & 0,471083 & 0,000169 & $1,38 €$ & $3,9021 \%$ & $0,09 €$ \\
\hline 71 & 116 & $10.000,00 €$ & $-€$ & 0,493923 & 0,000089 & $0,80 €$ & $3,9060 \%$ & $0,05 €$ \\
\hline 72 & 117 & $10.000,00 €$ & $-€$ & 0,517370 & 0,000045 & $0,44 €$ & $3,9099 \%$ & $0,03 €$ \\
\hline 73 & 118 & $10.000,00 €$ & $-€$ & 0,541424 & 0,000022 & $0,23 €$ & $3,9136 \%$ & $0,01 €$ \\
\hline 74 & 119 & $10.000,00 €$ & $-€$ & 0,566086 & 0,000010 & $0,12 €$ & $3,9173 \%$ & $0,01 €$ \\
\hline 75 & 120 & $10.000,00 €$ & $-€$ & 0,591354 & 0,000004 & $0,06 €$ & $3,9209 \%$ & $0,00 €$ \\
\hline 76 & 121 & $10.000,00 €$ & $-€$ & 0,617230 & 0,000002 & $0,03 €$ & $3,9244 \%$ & $0,00 €$ \\
\hline 77 & 122 & $10.000,00 €$ & $-€$ & 0,643713 & 0,000001 & $0,01 €$ & $3,9279 \%$ & $0,00 €$ \\
\hline 78 & 123 & $10.000,00 €$ & $-€$ & 0,670802 & 0,000000 & $0,00 €$ & $3,9312 \%$ & $0,00 €$ \\
\hline 79 & 124 & $10.000,00 €$ & $-€$ & 0,698499 & 0,000000 & $0,00 €$ & $3,9345 \%$ & $0,00 €$ \\
\hline 80 & 125 & $10.000,00 €$ & $-€$ & 0,726803 & 0,000000 & $0,00 €$ & $3,9377 \%$ & $0,00 €$ \\
\hline 81 & 126 & $10.000,00 €$ & $-€$ & 1,150000 & 0,000000 & $0,00 €$ & $3,9409 \%$ & $0,00 €$ \\
\hline
\end{tabular}

Fuente: Elaboración propia. 
